\title{
Three-Dimensional Biorthogonal Divergence-Free and Curl-Free Wavelets with Free-Slip Boundary
}

\author{
Yingchun Jiang and Qingqing Sun \\ School of Mathematics and Computational Science, Guilin University of Electronic Technology, Guilin 541004, China \\ Correspondence should be addressed to Yingchun Jiang; guilinjiang@126.com
}

Received 17 August 2013; Accepted 16 November 2013

Academic Editor: Antonio J. M. Ferreira

Copyright (C) 2013 Y. Jiang and Q. Sun. This is an open access article distributed under the Creative Commons Attribution License, which permits unrestricted use, distribution, and reproduction in any medium, provided the original work is properly cited.

\begin{abstract}
This paper deals with the construction of divergence-free and curl-free wavelets on the unit cube, which satisfies the free-slip boundary conditions. First, interval wavelets adapted to our construction are introduced. Then, we provide the biorthogonal divergence-free and curl-free wavelets with free-slip boundary and simple structure, based on the characterization of corresponding spaces. Moreover, the bases are also stable.
\end{abstract}

\section{Introduction}

In recent years, divergence-free and curl-free wavelets are generally studied, due to their potential use in many physical problems [1-5]. Anisotropic divergence-free and curl-free wavelets on the hypercube are firstly constructed in $[6,7]$, but all these functions only satisfy slip boundary conditions. However, the free-slip boundary is important in many cases, such as the solution of partial differential equations in incompressible fluids and electromagnetism. Inspired by this fact, $[8,9]$ give the construction of anisotropic divergencefree and curl-free wavelets with free-slip boundary, but the structure is very complicated and the basis functions are not explicit. Recently, based on a simple characterization of 2D divergence-free space, Harouna and Perrier proposed an alternative construction to [8] for divergence-free wavelets in two-dimensional case [10]. Following the similar but nontrivial line, we mainly study the anisotropic 3D divergencefree and curl-free wavelet bases with free-slip boundary in this paper. The traditional understanding that 3D curl-free wavelets are more difficult to construct than divergence-free wavelets is not always right, due to our procedure.

In Section 2, interval wavelets that we will use are introduced. Based on the spaces characterization, 3D biorthogonal divergence-free and curl-free wavelet bases are given in Sections 3 and 4 , respectively.

\section{Interval Wavelets on $[0,1]$}

In this part, we will introduce the interval wavelets used in the subsequent construction.

The existence of divergence-free and curl-free wavelets on $R^{d}$ follows from the following fundamental proposition [11].

Proposition 1. Let $\left(V_{j}^{1}(R), \widetilde{V}_{j}^{1}(R)\right)$ be a biorthogonal MRA of $L^{2}(R)$, with compactly supported scaling functions $\left(\varphi^{1}, \widetilde{\varphi}^{1}\right)$ and wavelets $\left(\psi^{1}, \widetilde{\psi}^{1}\right)$, such that $\varphi^{1}, \psi^{1} \in C^{1+\varepsilon}$ for $\varepsilon>0$. Then there exists a biorthogonal MRA $\left(V_{j}^{0}(R), \widetilde{V}_{j}^{0}(R)\right)$, with associated scaling functions $\left(\varphi^{0}, \widetilde{\varphi}^{0}\right)$ and wavelets $\left(\psi^{0}, \widetilde{\psi}^{0}\right)$, such that

$$
\left(\varphi^{1}\right)^{\prime}(x)=\varphi^{0}(x)-\varphi^{0}(x-1), \quad\left(\psi^{1}\right)^{\prime}=4 \psi^{0} .
$$

The dual functions verify $\int_{x}^{x+1} \widetilde{\varphi}^{1}(t) d t=\widetilde{\varphi}^{0}(x)$ and $\left(\widetilde{\psi}^{0}\right)^{\prime}=$ $-4 \widetilde{\psi}^{1}$.

Based on the above proposition, Jouini and LemariéRieusset [12] proved the existence of two one dimensional MRAs of $L^{2}(0,1)$ linked by

$$
\begin{gathered}
\frac{d}{d x} V_{j}^{1}=V_{j}^{0}, \\
\widetilde{V}_{j}^{0}=H_{0}^{1}(0,1) \bigcap \int_{0}^{x} \widetilde{V}_{j}^{1}=\left\{f: f^{\prime} \in \widetilde{V}_{j}^{1}, f(0)=f(1)=0\right\} .
\end{gathered}
$$


In the following, we simply introduce the construction of these spaces. Suppose that $\varphi^{1}$ in Proposition 1 is supported on $\left[n_{\min }, n_{\max }\right]\left(n_{\min }, n_{\max }\right.$ integers $)$ and reproduces polynomials up to degree $r-1$ :

$$
0 \leq \ell \leq r-1, \quad \frac{x^{\ell}}{\ell !}=\sum_{k=-\infty}^{+\infty} \tilde{p}_{\ell}^{1}(k) \varphi^{1}(x-k), \quad x \in R,
$$

with $\widetilde{p}_{\ell}^{1}(k)=\left\langle x^{\ell} / \ell !, \widetilde{\varphi}^{1}(x-k)\right\rangle$. Similarly, $\widetilde{\varphi}^{1}$ is supported on $\left[\widetilde{n}_{\min }, \widetilde{n}_{\max }\right]$ and reproduces polynomials up to degree $\widetilde{r}-1$.

For $j$ being sufficiently large, the spaces $V_{j}^{1}$ have the structure

$$
\begin{aligned}
V_{j}^{1}= & \operatorname{span}\left\{\Phi_{j, \ell}^{1, b}=2^{j / 2} \Phi_{\ell}^{1, b}\left(2^{j} x\right)\right\}_{\ell=0, \ldots, r-1} \oplus V_{j}^{1, \text { int }} \\
& \oplus \operatorname{span}\left\{\Phi_{j, \ell}^{1, \sharp}=2^{j / 2} \Phi_{\ell}^{1, \sharp}\left(2^{j} x\right)\right\}_{\ell=0, \ldots, r-1},
\end{aligned}
$$

where $V_{j}^{1, \text { int }}=\operatorname{span}\left\{\varphi_{j, k}^{1}=2^{j / 2} \varphi^{1}\left(2^{j} x-k\right): k=k_{b}, \ldots, 2^{j}-\right.$ $\left.k_{\sharp}\right\}$ is the space whose supports are included into $\left[\delta_{b} / 2^{j}, 1-\right.$ $\left.\delta_{\sharp} / 2^{j}\right] \subset[0,1]\left(\delta_{b}, \delta_{\sharp} \in N\right.$ be two fixed parameters), and $k_{b}=\delta_{b}-n_{\text {min }}, k_{\sharp}=\delta_{\sharp}+n_{\text {max }}$. Moreover, $\Phi_{\ell}^{1, b}$ are the edge scaling functions at the edge 0 being defined by

$$
\Phi_{\ell}^{1, b}(x)=\sum_{k=1-n_{\max }}^{k_{b}-1} \tilde{p}_{\ell}^{1}(k) \varphi^{1}(x-k) \chi_{[0,+\infty)} .
$$

At the edge $1, \Phi_{\ell}^{1, b}$ are defined by symmetry using $T f(x)=$ $f(1-x)$.

Similarly, the biorthogonal spaces $\widetilde{V}_{j}^{1}$ are defined with the same structure as

$$
\widetilde{V}_{j}^{1}=\operatorname{span}\left\{\widetilde{\Phi}_{j, \ell}^{1, b}\right\}_{\ell=0, \ldots, \widetilde{r}-1} \oplus \widetilde{V}_{j}^{1, \text { int }} \oplus \operatorname{span}\left\{\widetilde{\Phi}_{j, \ell}^{1, \sharp}\right\}_{\ell=0, \ldots, \widetilde{r}-1} .
$$

Adjusting the parameters such that

$$
\begin{aligned}
\Delta_{j} & =\operatorname{dim}\left(V_{j}^{1}\right)=\operatorname{dim}\left(\widetilde{V}_{j}^{1}\right) \\
& =2^{j}-\left(\delta_{b+\delta_{\sharp}}\right)-\left(n_{\max }-n_{\min }\right)+2 r+1 .
\end{aligned}
$$

The last step of the construction is the biorthogonalization process, since the edge scaling functions of $V_{j}^{1}$ and $\widetilde{V}_{j}^{1}$ are no more biorthogonal. Finally, $\left(V_{j}^{1}, \widetilde{V}_{j}^{1}\right)$ form a biorthogonal MRA of $L^{2}(0,1)$.

As described in [13], removing the edge scaling functions $\Phi_{0}^{1, b}$ and $\Phi_{0}^{1, \sharp}$ leads to

$$
\begin{aligned}
V_{j}^{D} & =\operatorname{span}\left\{\Phi_{j, \ell}^{1, b}\right\}_{\ell=1, \ldots, r-1} \oplus V_{j}^{1, \text { int }} \oplus \operatorname{span}\left\{\Phi_{j, \ell}^{1, \sharp}\right\}_{\ell=1, \ldots, r-1} \\
& =: \operatorname{span}\left\{\varphi_{j, k}^{D}: k=1, \ldots, \Delta_{j}-2\right\} .
\end{aligned}
$$

Similarly, define $\widetilde{V}_{j}^{D}=\operatorname{span}\left\{\widetilde{\Phi}_{j, \ell}^{1, b}\right\}_{\ell=1, \ldots, \tilde{r}_{-1}} \oplus \widetilde{V}_{j}^{1, \text { int }} \oplus$ $\operatorname{span}\left\{\widetilde{\Phi}_{j, \ell}^{1, \sharp}\right\}_{\ell=1, \ldots, \tilde{r}-1}$. After a biorthogonalization process, we finally note that

$$
\widetilde{V}_{j}^{D}=\operatorname{span}\left\{\widetilde{\varphi}_{j, k}^{D}: k=1, \ldots, \Delta_{j}-2\right\},
$$

and the spaces $\left(V_{j}^{D}, \widetilde{V}_{j}^{D}\right)$ form a biorthogonal MRA of $H_{0}^{1}(0$, 1).

The construction of $\left(V_{j}^{0}, \widetilde{V}_{j}^{0}\right)$ follows the same structure. Since $\left(\varphi^{1}\right)^{\prime}(x)=\varphi^{0}(x)-\varphi^{0}(x-1), \varphi^{0}$ has compact support $\left[n_{\min }, n_{\max }-1\right]$ and reproduces polynomials up to degree $r-2$ :

$$
0 \leq \ell \leq r-2, \quad \frac{x^{\ell}}{\ell !}=\sum_{k=-\infty}^{+\infty} \widetilde{p}_{\ell}^{0}(k) \varphi^{0}(x-k),
$$

with $\widetilde{p}_{\ell}^{0}(k)=\left\langle x^{\ell} / \ell !, \widetilde{\varphi}^{0}(x-k)\right\rangle$. The scaling function $\widetilde{\varphi}^{0}(x)=$ $\int_{x}^{x+1} \widetilde{\varphi}^{1}(t) d t$ has support $\left[\widetilde{n}_{\min }-1, \widetilde{n}_{\max }\right]$ and reproduces polynomials up to degree $\widetilde{r}$. Consider

$$
\begin{aligned}
V_{j}^{0}= & \operatorname{span}\left\{\Phi_{j, \ell}^{0, b}=2^{j / 2} \Phi_{\ell}^{0, b}\left(2^{j} x\right)\right\}_{\ell=0, \ldots, r-2} \oplus V_{j}^{0, \text { int }} \\
& \oplus \operatorname{span}\left\{\Phi_{j, \ell}^{0, \sharp}=2^{j / 2} \Phi_{\ell}^{0, \sharp}\left(2^{j} x\right)\right\}_{\ell=0, \ldots, r-2},
\end{aligned}
$$

where $V_{j}^{0, \text { int }}=\operatorname{span}\left\{\varphi_{j, k}^{0}=2^{j / 2} \varphi^{0}\left(2^{j} x-k\right): k=k_{b}, \ldots, 2^{j}-\right.$ $\left.k_{\sharp}+1\right\}$ and supports are included into $\left[\delta_{b} / 2^{j}, 1-\delta_{\sharp} / 2^{j}\right] \subset$ $[0,1]$. The left edge scaling functions are

$$
\Phi_{\ell}^{0, b}(x)=\sum_{k=2-n_{\max }}^{k_{\mathrm{b}}-1} \widetilde{p}_{\ell}^{0}(k) \varphi^{0}(x-k) \chi_{[0,+\infty)} .
$$

Biorthogonal spaces $\widetilde{V}_{j}^{0}$ are similarly defined, but by satisfying vanishing boundary conditions at 0 and 1 , then

$$
\widetilde{V}_{j}^{0}=\operatorname{span}\left\{\widetilde{\Phi}_{j, \ell}^{0, b}\right\}_{\ell=1, \ldots, \widetilde{r}} \oplus \widetilde{V}_{j}^{0, \text { int }} \oplus \operatorname{span}\left\{\widetilde{\Phi}_{j, \ell}^{0, \sharp}\right\}_{\ell=1, \ldots, \widetilde{r}},
$$

with $\widetilde{V}_{j}^{0, \text { int }}=\operatorname{span}\left\{\widetilde{\varphi}_{j, k}^{0}: k=\widetilde{k}_{b}+1, \ldots, 2^{j}-\widetilde{k}_{\sharp}\right\}$ and $\widetilde{\Phi}_{\ell}^{0, b}=$ $\sum_{k=1-\widetilde{n}_{\max }}^{\widetilde{k}_{b}} p_{\ell}^{0}(k) \widetilde{\varphi}^{0}(x-k) \chi_{[0,+\infty)}$ for $\ell=1, \ldots, \widetilde{r}$. It is easy to know $\operatorname{dim}\left(V_{j}^{0}\right)=\operatorname{dim}\left(\widetilde{V}_{j}^{0}\right)=\Delta_{j}-1$.

In practice, we choose $j \geq j_{\text {min }}$ with

$$
\begin{aligned}
j_{\text {min }}>\max \left\{\log _{2}\left[n_{\text {max }}-n_{\text {min }}+\delta_{\sharp}+\delta_{b}+1\right],\right. \\
\left.\log _{2}\left[\widetilde{n}_{\text {max }}-\widetilde{n}_{\text {min }}+\widetilde{\delta}_{\sharp}+\widetilde{\delta}_{b}+1\right]\right\}
\end{aligned}
$$

to ensure that the supports of edge scaling functions at 0 do not intersect the supports of edge scaling functions at 1.

The construction of wavelet spaces $\left(W_{j}^{1}, \widetilde{W}_{j}^{1}\right)$ can be seen from [13]. Moreover, they satisfy the following result.

Proposition 2 (see [12]). Let $\left(V_{j}^{1}, \widetilde{V}_{j}^{1}\right)$ and $\left(V_{j}^{0}, \widetilde{V}_{j}^{0}\right)$ be MRAs satisfying $(d / d x) V_{j}^{1}=V_{j}^{0}$ and $\widetilde{V}_{j}^{0}=H_{0}^{1} \cap \int_{0}^{x} \widetilde{V}_{j}^{1}$; then the wavelet spaces $W_{j}^{0}$ and $\widetilde{W}_{j}^{0}$ are linked to the biorthogonal wavelet spaces associated to $\left(V_{j}^{1}, \widetilde{V}_{j}^{1}\right)$ by

$$
W_{j}^{0}=\frac{d}{d x} W_{j}^{1}, \quad \widetilde{W}_{j}^{0}=\int_{0}^{x} \widetilde{W}_{j}^{1} .
$$

Moreover, let $\left\{\psi_{j, k}^{1}\right\}_{k=1, \ldots, 2^{j}}$ and $\left\{\widetilde{\psi}_{j, k}^{1}\right\}_{k=1, \ldots, 2^{j}}$ be two biorthogonal wavelet bases of $W_{j}^{1}$ and $\widetilde{W}_{j}^{1}$. Biorthogonal wavelet bases of $W_{j}^{0}$ and $\widetilde{W}_{j}^{0}$ are directly defined by

$$
\psi_{j, k}^{0}=2^{-j}\left(\psi_{j, k}^{1}\right)^{\prime}, \quad \widetilde{\psi}_{j, k}^{0}=-2^{j} \int_{0}^{x} \widetilde{\psi}_{j, k}^{1} .
$$




\section{Divergence-Free Wavelets on $[0,1]^{3}$}

Let $\Omega=[0,1]^{3}$ and let $\vec{n}$ be the normal vector; the boundary condition considered in [6] is $\vec{u} \cdot \vec{n}=0$ on $\Gamma=\bigcup_{k=1}^{3} \Gamma_{k}$ with

$$
\Gamma_{k}=[0,1]^{k-1} \times\{0\} \times[0,1]^{3-k}, \quad 1 \leq k \leq 3 .
$$

It holds that $\vec{u} \cdot \vec{n}=0$ on $\Gamma$ if and only if $u_{k}=0$ on $\Gamma_{k}(1 \leq k \leq$ $3)$. We call it a slip boundary, which is shown in Figure 1.

In this section, we mainly consider the following space with free-slip boundary as Figure 2

$$
\mathscr{H}_{\operatorname{div}}(\Omega)=\left\{\vec{u} \in\left(L^{2}(\Omega)\right)^{3}: \operatorname{div} \vec{u}=0, \vec{u} \cdot \vec{n}=0 \text { on } \partial \Omega\right\} .
$$

For $\vec{u}(x, y, z)=\left(u_{1}, u_{2}, u_{3}\right)^{T}$, the $3 \mathrm{D}$ curl-operator is defined as

$$
\operatorname{curl} \vec{u}=\left(\partial_{2} u_{3}-\partial_{3} u_{2}, \partial_{3} u_{1}-\partial_{1} u_{3}, \partial_{1} u_{2}-\partial_{2} u_{1}\right)^{T} .
$$

Remark 3. Taking Fourier transform on the both sides of $\operatorname{div} \vec{u}=0$ leads to the equation

$$
\xi_{1} \widehat{u}_{1}(\xi)+\xi_{2} \widehat{u}_{2}(\xi)+\xi_{3} \widehat{u}_{3}(\xi)=0, \quad \xi=\left(\xi_{1}, \xi_{2}, \xi_{3}\right) .
$$

In $L^{2}\left(R^{3}\right)$, define the following functions

$$
\begin{gathered}
\widehat{\varphi}_{1}(\xi)=\frac{\xi_{3} \widehat{u}_{2}-\xi_{2} \widehat{u}_{3}}{i\left(\xi_{1}^{2}+\xi_{2}^{2}+\xi_{3}^{2}\right)}, \quad \widehat{\varphi}_{2}(\xi)=\frac{\xi_{1} \widehat{u}_{3}-\xi_{3} \widehat{u}_{1}}{i\left(\xi_{1}^{2}+\xi_{2}^{2}+\xi_{3}^{2}\right)}, \\
\widehat{\varphi}_{3}(\xi)=\frac{\xi_{2} \widehat{u}_{1}-\xi_{1} \widehat{u}_{2}}{i\left(\xi_{1}^{2}+\xi_{2}^{2}+\xi_{3}^{2}\right)} .
\end{gathered}
$$

Then, according to (20), it is easy to verify that

$$
\begin{aligned}
& \widehat{u}_{1}(\xi)=i\left(\xi_{2} \widehat{\varphi}_{3}(\xi)-\xi_{3} \widehat{\varphi}_{2}(\xi)\right), \\
& \widehat{u}_{2}(\xi)=i\left(\xi_{3} \widehat{\varphi}_{1}(\xi)-\xi_{1} \widehat{\varphi}_{3}(\xi)\right), \\
& \widehat{u}_{3}(\xi)=i\left(\xi_{1} \widehat{\varphi}_{2}(\xi)-\xi_{2} \widehat{\varphi}_{1}(\xi)\right),
\end{aligned}
$$

which is equivalent to $\vec{u}=\operatorname{curl} \vec{\varphi}$. Therefore, any function $\vec{u} \in\left(L^{2}\left(R^{3}\right)\right)^{3}$ which satisfies $\operatorname{div} \vec{u}=0$ can be characterized by curl operator as $\vec{u}=\operatorname{curl} \vec{\varphi}$ with $\vec{\varphi} \in\left(H^{1}\left(R^{3}\right)\right)^{3}$. In fact, a similar result holds in 3D nonsmooth domains.

Proposition 4 (see [14]). There is a characterization

$$
\begin{aligned}
\mathscr{H}_{\text {div }} & (\Omega) \\
& =\left\{\vec{u}=\operatorname{curl} \vec{\varphi}: \vec{\varphi} \in\left(H^{1}(\Omega)\right)^{3}, \vec{\varphi} \times \vec{n}=\overrightarrow{0} \text { on } \partial \Omega\right\} .
\end{aligned}
$$

Based on Proposition 4, we give the following definition of divergence-free scaling function spaces.
Definition 5. For $j \geq j_{\min }$, the divergence-free scaling function spaces $\vec{V}_{j}^{\text {div }}$ are defined by

$$
\begin{aligned}
\vec{V}_{j}^{\mathrm{div}}=\operatorname{curl} & \left\{\left(V_{j}^{0} \otimes V_{j}^{D} \otimes V_{j}^{D}\right) \times\left(V_{j}^{D} \otimes V_{j}^{0} \otimes V_{j}^{D}\right)\right. \\
& \left.\times\left(V_{j}^{D} \otimes V_{j}^{D} \otimes V_{j}^{0}\right)\right\} \\
=\operatorname{span} & \left\{\Phi_{j, \mathbf{k}}^{\mathrm{div}, 1}, \Phi_{j, \mathbf{k}}^{\mathrm{div}, 2}, \Phi_{j, \mathbf{k}}^{\mathrm{div}, 3}\right\},
\end{aligned}
$$

where the divergence-free scaling functions are given by

$$
\begin{aligned}
\Phi_{j, \mathbf{k}}^{\mathrm{div}, 1} & =: \frac{1}{\sqrt{2}} \operatorname{curl}\left[\left(\varphi_{j, k_{1}}^{0} \cdot \varphi_{j, k_{2}}^{D} \cdot \varphi_{j, k_{3}}^{D}, 0,0\right)^{T}\right] \\
& =\frac{1}{\sqrt{2}}\left[\varphi_{j, k_{1}}^{0} \cdot \varphi_{j, k_{2}}^{D} \cdot\left(\varphi_{j, k_{3}}^{D}\right)^{\prime} \delta_{2}-\varphi_{j, k_{1}}^{0} \cdot\left(\varphi_{j, k_{2}}^{D}\right)^{\prime} \cdot \varphi_{j, k_{3}}^{D} \delta_{3}\right], \\
\Phi_{j, \mathbf{k}}^{\mathrm{div}, 2} & =: \frac{1}{\sqrt{2}} \operatorname{curl}\left[\left(0, \varphi_{j, k_{1}}^{D} \cdot \varphi_{j, k_{2}}^{0} \cdot \varphi_{j, k_{3}}^{D}, 0\right)^{T}\right] \\
& =\frac{1}{\sqrt{2}}\left[\left(\varphi_{j, k_{1}}^{D}\right)^{\prime} \cdot \varphi_{j, k_{2}}^{0} \cdot \varphi_{j, k_{3}}^{D} \delta_{3}-\varphi_{j, k_{1}}^{D} \cdot \varphi_{j, k_{2}}^{0} \cdot\left(\varphi_{j, k_{3}}^{D}\right)^{\prime} \delta_{1}\right], \\
\Phi_{j, \mathbf{k}}^{\mathrm{div}, 3} & =: \frac{1}{\sqrt{2}} \operatorname{curl}\left[\left(0,0, \varphi_{j, k_{1}}^{D} \cdot \varphi_{j, k_{2}}^{D} \cdot \varphi_{j, k_{3}}^{0}\right)^{T}\right] \\
& =\frac{1}{\sqrt{2}}\left[\varphi_{j, k_{1}}^{D} \cdot\left(\varphi_{j, k_{2}}^{D}\right)^{\prime} \cdot \varphi_{j, k_{3}}^{0} \delta_{1}-\left(\varphi_{j, k_{1}}^{D}\right)^{\prime} \cdot \varphi_{j, k_{2}}^{D} \cdot \varphi_{j, k_{3}}^{0} \delta_{2}\right] .
\end{aligned}
$$

For proving the consequent main result, we also consider the standard MRA $\vec{V}_{j}$ of $\left(L^{2}(\Omega)\right)^{3}$ :

$$
\begin{aligned}
\vec{V}_{j}= & \left(V_{j}^{1} \otimes V_{j}^{0} \otimes V_{j}^{0}\right) \times\left(V_{j}^{0} \otimes V_{j}^{1} \otimes V_{j}^{0}\right) \\
& \times\left(V_{j}^{0} \otimes V_{j}^{0} \otimes V_{j}^{1}\right) .
\end{aligned}
$$

The following conclusion shows that the space $\vec{V}_{j}$ preserves the divergence-free condition.

Proposition 6. If $\vec{u} \in\left(L^{2}(\Omega)\right)^{3}$ and $\operatorname{div} \vec{u}=0$, then $\operatorname{div}\left[\vec{P}_{j} \vec{u}\right]=$ 0 , where $\vec{P}_{j}=\left(p_{j}^{1} \otimes p_{j}^{0} \otimes p_{j}^{0}, p_{j}^{0} \otimes p_{j}^{1} \otimes p_{j}^{0}, p_{j}^{0} \otimes p_{j}^{0} \otimes p_{j}^{1}\right)$ is the biorthogonal projector on $\vec{V}_{j}$.

Proof. Let $\vec{u}=\left(u_{1}, u_{2}, u_{3}\right)^{T}$; then

$$
\vec{P}_{j} \vec{u}=\left(p_{j}^{1} \otimes p_{j}^{0} \otimes p_{j}^{0} u_{1}, p_{j}^{0} \otimes p_{j}^{1} \otimes p_{j}^{0} u_{2}, p_{j}^{0} \otimes p_{j}^{0} \otimes p_{j}^{1} u_{3}\right)^{T} .
$$

Therefore, by the fact $d / d x \circ p_{j}^{1} f=p_{j}^{0} \circ(d / d x) f$ in [10], we obtain

$$
\begin{aligned}
\operatorname{div}\left[\vec{P}_{j} \vec{u}\right]= & \frac{\partial}{\partial x} p_{j}^{1} \otimes p_{j}^{0} \otimes p_{j}^{0} u_{1}+\frac{\partial}{\partial y} p_{j}^{0} \otimes p_{j}^{1} \otimes p_{j}^{0} u_{2} \\
& +\frac{\partial}{\partial z} p_{j}^{0} \otimes p_{j}^{0} \otimes p_{j}^{1} u_{3} \\
= & p_{j}^{0} \otimes p_{j}^{0} \otimes p_{j}^{0}\left(\frac{\partial u_{1}}{\partial x}+\frac{\partial u_{2}}{\partial y}+\frac{\partial u_{3}}{\partial z}\right) \\
= & p_{j}^{0} \otimes p_{j}^{0} \otimes p_{j}^{0}(\operatorname{div} \vec{u})=0 .
\end{aligned}
$$



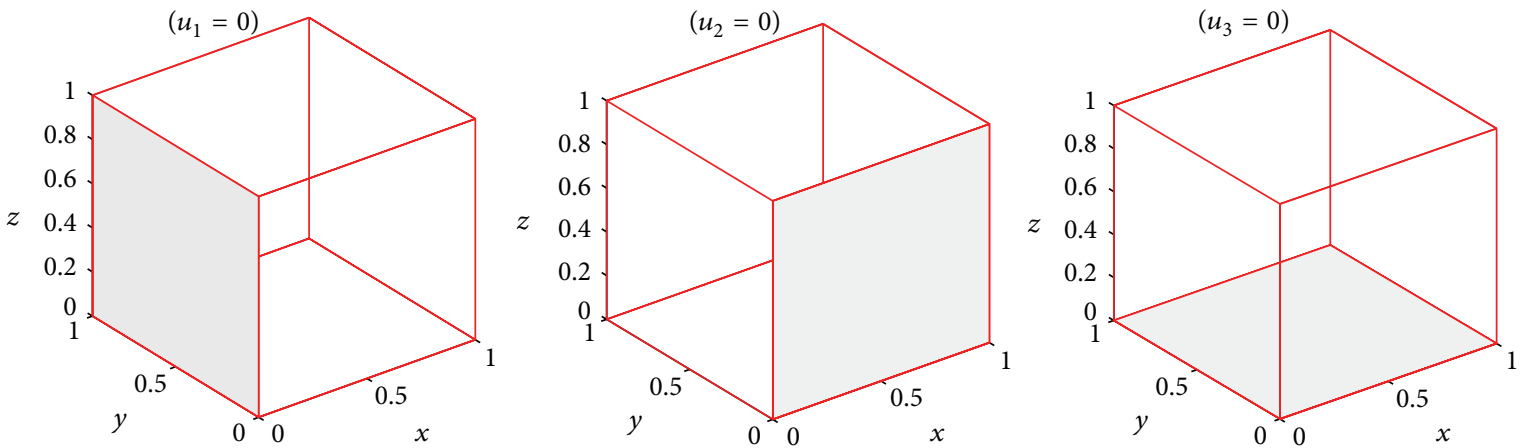

FIGURE 1: Slip boundary condition (divergence).
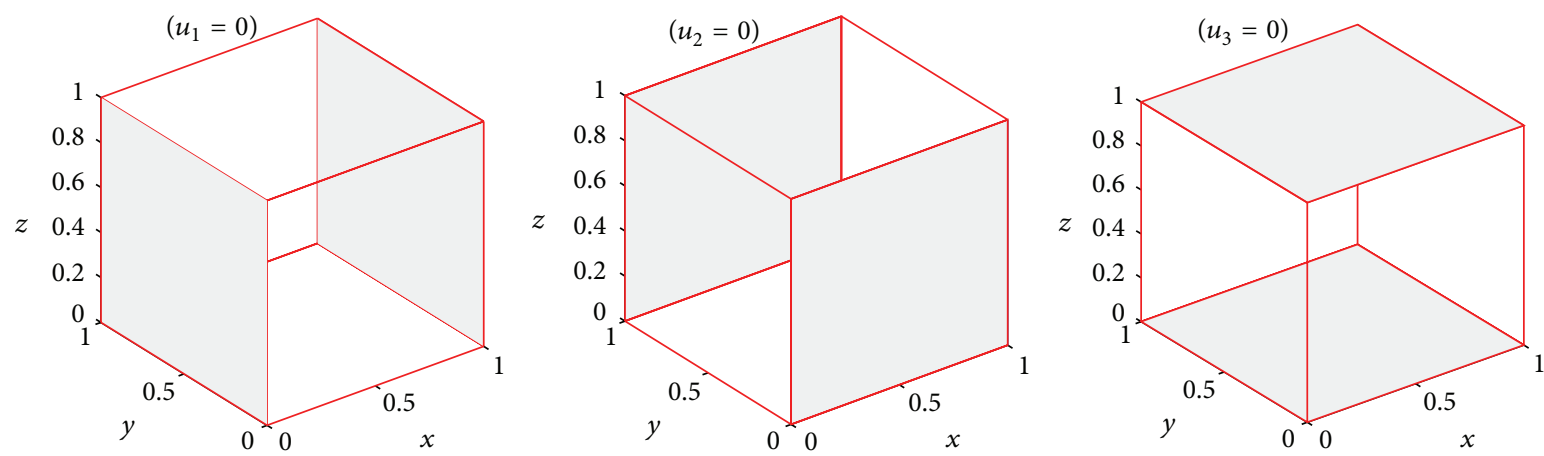

Figure 2: Free-slip boundary condition (divergence).

Theorem 7. The divergence-free scaling function spaces $\left\{\vec{V}_{j}^{\mathrm{div}}\right\}_{j \geq j_{\min }}$ is a multiresolution analysis of $\mathscr{H}_{\mathrm{div}}(\Omega)$.

Proof. Since $\mathscr{H}_{\text {div }}(\Omega) \cap \vec{V}_{j}$ are a multiresolution analysis of $\mathscr{H}_{\text {div }}(\Omega)$, it is reduced to prove

$$
\vec{V}_{j}^{\text {div }}=\mathscr{H}_{\text {div }}(\Omega) \cap \vec{V}_{j}
$$

Noting that $(d / d x) V_{j}^{1}=V_{j}^{0}$ and $V_{j}^{D} \subseteq V_{j}^{1}$ from (2) and (8), we know $\vec{V}_{j}^{\text {div }} \subset \vec{V}_{j}$. Furthermore, $\vec{V}_{j}^{\text {div }} \subset \mathscr{H}_{\text {div }}(\Omega)$ by construction. Therefore, $\vec{V}_{j}^{\text {div }} \subset \mathscr{H}_{\text {div }}(\Omega) \cap \vec{V}_{j}$.

Conversely, letting $\vec{u} \in \mathscr{H}_{\text {div }}(\Omega) \cap \vec{V}_{j}$, we are going to prove $\vec{u} \in \vec{V}_{j}^{\text {div }}$. On the one hand, since $\vec{u} \in \vec{V}_{j}$, we have $\vec{u}=\vec{P}_{j} \vec{u}$. On the other hand, since $\vec{u} \in \mathscr{H}_{\text {div }}(\Omega)$, there exists a $\vec{\varphi}=$ $\left(\varphi_{1}, \varphi_{2}, \varphi_{3}\right)^{T} \in\left(H^{1}(\Omega)\right)^{3}$ such that $\vec{u}=\operatorname{curl}(\vec{\varphi})$. Moreover, $\vec{\varphi} \times \vec{n}=\overrightarrow{0}$. Thus, $\vec{u}=\vec{P}_{j}[\operatorname{curl}(\vec{\varphi})]$. Furthermore, we can decompose $\vec{\varphi}$ by isotropic vector wavelets as

$$
\vec{\varphi}=\vec{P}_{j}^{D}(\vec{\varphi})+\sum_{j^{\prime} \geq j} \vec{Q}_{j^{\prime}}^{D}(\vec{\varphi})
$$

where $\vec{P}_{j}^{D}(\vec{\varphi})=\sum_{k} c_{1, k} \varphi_{j, k_{1}}^{0} \varphi_{j, k_{2}}^{D} \varphi_{j, k_{3}}^{D} \delta_{1}+\sum_{k} c_{2, k} \varphi_{j, k_{1}}^{D} \varphi_{j, k_{2}}^{0} \varphi_{j, k_{3}}^{D}$ $\delta_{2}+\sum_{k} c_{3, k} \varphi_{j, k_{1}}^{D} \varphi_{j, k_{2}}^{D} \varphi_{j, k_{3}}^{0} \delta_{3}$

$$
\begin{aligned}
\vec{Q}_{j^{\prime}}^{D}(\vec{\varphi}) & \sum_{k}\left(d_{j^{\prime}, k}^{1,1} \varphi_{j^{\prime}, k_{1}}^{0} \varphi_{j^{\prime}, k_{2}}^{D} \psi_{j^{\prime}, k_{3}}^{D}+d_{j^{\prime}, k}^{1,2} \varphi_{j^{\prime}, k_{1}}^{0} \psi_{j^{\prime}, k_{2}}^{D} \varphi_{j^{\prime}, k_{3}}^{D}\right. \\
+ & d_{j^{\prime}, k}^{1,3} \psi_{j^{\prime}, k_{1}}^{0} \varphi_{j^{\prime}, k_{2}}^{D} \varphi_{j^{\prime}, k_{3}}^{D}+d_{j^{\prime}, k}^{1,4} \varphi_{j^{\prime}, k_{1}}^{0} \psi_{j^{\prime}, k_{2}}^{D} \psi_{j^{\prime}, k_{3}}^{D} \\
+ & d_{j^{\prime}, k}^{1,5} \psi_{j^{\prime}, k_{1}}^{0} \varphi_{j^{\prime}, k_{2}}^{D} \psi_{j^{\prime}, k_{3}}^{D}+d_{j^{\prime}, k}^{1,6} \psi_{j^{\prime}, k_{1}}^{0} \psi_{j^{\prime}, k_{2}}^{D} \varphi_{j^{\prime}, k_{3}}^{D} \\
+ & \left.d_{j^{\prime}, k}^{1,7} \psi_{j^{\prime}, k_{1}}^{0} \psi_{j^{\prime}, k_{2}}^{D} \psi_{j^{\prime}, k_{3}}^{D}\right) \delta_{1} \\
+\sum_{k}( & d_{j^{\prime}, k}^{2,1} \varphi_{j^{\prime}, k_{1}}^{D} \varphi_{j^{\prime}, k_{2}}^{0} \psi_{j^{\prime}, k_{3}}^{D}+d_{j^{\prime}, k}^{2,2} \varphi_{j^{\prime}, k_{1}}^{D} \psi_{j^{\prime}, k_{2}}^{0} \varphi_{j^{\prime}, k_{3}}^{D} \\
& +d_{j^{\prime}, k}^{2,3} \psi_{j^{\prime}, k_{1}}^{D} \varphi_{j^{\prime}, k_{2}}^{0} \varphi_{j^{\prime}, k_{3}}^{D}+d_{j^{\prime}, k}^{2,4} \varphi_{j^{\prime}, k_{1}}^{D} \psi_{j^{\prime}, k_{2}}^{0} \psi_{j^{\prime}, k_{3}}^{D} \\
& +d_{j^{\prime}, k}^{2,5} \psi_{j^{\prime}, k_{1}}^{D} \varphi_{j^{\prime}, k_{2}}^{0} \psi_{j^{\prime}, k_{3}}^{D}+d_{j^{\prime}, k}^{2,6} \psi_{j^{\prime}, k_{1}}^{D} \psi_{j^{\prime}, k_{2}}^{0} \varphi_{j^{\prime}, k_{3}}^{D} \\
& \left.+d_{j^{\prime}, k}^{2,7} \psi_{j^{\prime}, k_{1}}^{D} \psi_{j^{\prime}, k_{2}}^{0} \psi_{j^{\prime}, k_{3}}^{D}\right) \delta_{2} \\
& \left.+d_{j^{\prime}, k}^{3,7} \psi_{j^{\prime}, k_{1}}^{D} \psi_{j^{\prime}, k_{2}}^{D} \psi_{j^{\prime}, k_{3}}^{0}\right) \delta_{3} . \\
& +d_{j^{\prime}, k}^{3,1} \varphi_{j^{\prime}, k_{1}}^{D} \varphi_{j^{\prime}, k_{2}}^{D} \psi_{j^{\prime}, k_{3}}^{0}+d_{j^{\prime}, k}^{3,2} \varphi_{j^{\prime}, k_{1}}^{D} \psi_{j^{\prime}, k_{2}}^{D} \varphi_{j^{\prime}, k_{3}}^{0} \\
& +d_{j^{\prime}, k}^{3,3} \psi_{j^{\prime}, k_{1}}^{D} \varphi_{j^{\prime}, k_{2}}^{D} \varphi_{j^{\prime}, k_{3}}^{0}+d_{j^{\prime}, k}^{3,4} \varphi_{j^{\prime}, k_{1}}^{D} \psi_{j^{\prime}, k_{2}}^{D} \psi_{j^{\prime}, k_{3}}^{0} \\
& +d_{j^{\prime}, k}^{3,5} \psi_{j^{\prime}, k_{1}}^{D} \varphi_{j^{\prime}, k_{2}}^{D} \psi_{j^{\prime}, k_{3}}^{0}+d_{j^{\prime}, k}^{3,6} \psi_{j^{\prime}, k_{1}}^{D} \psi_{j^{\prime}, k_{2}}^{D} \varphi_{j^{\prime}, k_{3}}^{0} \\
+ &
\end{aligned}
$$


Since curl $\left[\varphi_{j^{\prime}, k_{1}}^{0} \varphi_{j^{\prime}, k_{2}}^{D} \psi_{j^{\prime}, k_{3}}^{D} \delta_{1}\right] \in\left(V_{j^{\prime}}^{D} \otimes V_{j^{\prime}}^{0} \otimes W_{j^{\prime}}^{0}\right) \times\left(V_{j^{\prime}}^{0} \otimes V_{j^{\prime}}^{D} \otimes\right.$ $\left.W_{j^{\prime}}^{0}\right) \times\left(V_{j^{\prime}}^{0} \otimes V_{j^{\prime}}^{0} \otimes W_{j^{\prime}}^{D}\right)$, then

$$
\vec{P}_{j}\left[\operatorname{curl}\left(\varphi_{j^{\prime}, k_{1}}^{0} \varphi_{j^{\prime}, k_{2}}^{D} \psi_{j^{\prime}, k_{3}}^{D} \delta_{1}\right)\right]=\overrightarrow{0} .
$$

Similarly, every term in the right sides of (31) satisfies (32). Finally,

$$
\vec{P}_{j}\left[\operatorname{curl}\left(\vec{Q}_{j^{\prime}}^{D}(\vec{\varphi})\right)\right]=\overrightarrow{0} .
$$

Furthermore, we can obtain

$$
\vec{u}=\vec{P}_{j}[\operatorname{curl}(\vec{\varphi})]=\vec{P}_{j}\left[\operatorname{curl}\left(\vec{P}_{j}^{D} \vec{\varphi}\right)\right]=\operatorname{curl}\left(\vec{P}_{j}^{D} \vec{\varphi}\right) .
$$

Here, we have used the fact $\operatorname{curl}\left(\vec{P}_{j}^{D} \vec{\varphi}\right) \in \vec{V}_{j}$ in the last step of (34). By construction, we have $\operatorname{curl}\left(\vec{P}_{j}^{D} \vec{\varphi}\right) \in \vec{V}_{j}^{\text {div }}$, which means $\vec{u} \in \vec{V}_{j}^{\text {div }}$ and the proof is completed.

Based on the constructive method of vector wavelets and the following decompositions:

$$
\begin{aligned}
& V_{j}^{0} \otimes V_{j}^{D} \otimes V_{j}^{D}=\left(V_{j_{\min }}^{0} \oplus_{j_{1}=j_{\min }}^{j-1} W_{j_{1}}^{0}\right) \\
& \otimes\left(V_{j_{\min }}^{D} \oplus_{j_{2}=j_{\min }}^{j-1} W_{j_{2}}^{D}\right) \\
& \otimes\left(V_{j_{\min }}^{D} \oplus_{j_{3}=j_{\min }}^{j-1} W_{j_{3}}^{D}\right) \\
& =\left(V_{j_{\min }}^{0} \otimes V_{j_{\min }}^{D} \otimes V_{j_{\min }}^{D}\right) \\
& \oplus_{j_{3}=j_{\min }}^{j-1}\left(V_{j_{\min }}^{0} \otimes V_{j_{\min }}^{D} \otimes W_{j_{3}}^{D}\right) \\
& \oplus_{j_{2}=j_{\min }}^{j-1}\left(V_{j_{\min }}^{0} \otimes W_{j_{2}}^{D} \otimes V_{j_{\min }}^{D}\right) \\
& \oplus_{j_{1}=j_{\min }}^{j-1}\left(W_{j_{1}}^{0} \otimes V_{j_{\min }}^{D} \otimes V_{j_{\min }}^{D}\right) \\
& \oplus_{j_{2}, j_{3}=j_{\min }}^{j-1}\left(V_{j_{\min }}^{0} \otimes W_{j_{2}}^{D} \otimes W_{j_{3}}^{D}\right) \\
& \oplus_{j_{1}, j_{3}=j_{\min }}^{j-1}\left(W_{j_{1}}^{0} \otimes V_{j_{\min }}^{D} \otimes W_{j_{3}}^{D}\right) \\
& \oplus_{j_{1}, j_{2}=j_{\min }}^{j-1}\left(W_{j_{1}}^{0} \otimes W_{j_{2}}^{D} \otimes V_{j_{\min }}^{D}\right) \\
& \oplus_{j_{1}, j_{2}, j_{3}=j_{\min }}^{j-1}\left(W_{j_{1}}^{0} \otimes W_{j_{2}}^{D} \otimes W_{j_{3}}^{D}\right), \\
& V_{j}^{D} \otimes V_{j}^{0} \otimes V_{j}^{D}=\left(V_{j_{\min }}^{D} \otimes V_{j_{\min }}^{0} \otimes V_{j_{\min }}^{D}\right) \\
& \oplus_{j_{3}=j_{\min }}^{j-1}\left(V_{j_{\min }}^{D} \otimes V_{j_{\min }}^{0} \otimes W_{j_{3}}^{D}\right) \\
& \oplus_{j_{2}=j_{\min }}^{j-1}\left(V_{j_{\min }}^{D} \otimes W_{j_{2}}^{0} \otimes V_{j_{\min }}^{D}\right) \\
& \oplus_{j_{1}=j_{\min }}^{j-1}\left(W_{j_{1}}^{D} \otimes V_{j_{\min }}^{0} \otimes V_{j_{\min }}^{D}\right) \\
& \oplus_{j_{2}, j_{3}=j_{\min }}^{j-1}\left(V_{j_{\min }}^{D} \otimes W_{j_{2}}^{0} \otimes W_{j_{3}}^{D}\right) \\
& \oplus_{j_{1}, j_{3}=j_{\min }}^{j-1}\left(W_{j_{1}}^{D} \otimes V_{j_{\text {min }}}^{0} \otimes W_{j_{3}}^{D}\right) \\
& \oplus_{j_{1}, j_{2}=j_{\min }}^{j-1}\left(W_{j_{1}}^{D} \otimes W_{j_{2}}^{0} \otimes V_{j_{\min }}^{D}\right) \\
& \oplus_{j_{1}, j_{2}, j_{3}=j_{\min }}^{j-1}\left(W_{j_{1}}^{D} \otimes W_{j_{2}}^{0} \otimes W_{j_{3}}^{D}\right) \text {, }
\end{aligned}
$$

$$
\begin{aligned}
V_{j}^{D} \otimes V_{j}^{D} \otimes V_{j}^{0}= & \left(V_{j_{\min }}^{D} \otimes V_{j_{\min }}^{D} \otimes V_{j_{\min }}^{0}\right) \\
& \oplus_{j_{3}=j_{\min }}^{j-1}\left(V_{j_{\min }}^{D} \otimes V_{j_{\min }}^{D} \otimes W_{j_{3}}^{0}\right) \\
& \oplus_{j_{2}=j_{\min }}^{j-1}\left(V_{j_{\min }}^{D} \otimes W_{j_{2}}^{D} \otimes V_{j_{\min }}^{0}\right) \\
& \oplus_{j_{1}=j_{\min }}^{j-1}\left(W_{j_{1}}^{D} \otimes V_{j_{\min }}^{D} \otimes V_{j_{\min }}^{0}\right) \\
& \oplus_{j_{2}, j_{3}=j_{\min }}^{j-1}\left(V_{j_{\min }}^{D} \otimes W_{j_{2}}^{D} \otimes W_{j_{3}}^{0}\right) \\
& \oplus_{j_{1}, j_{3}=j_{\min }}^{j-1}\left(W_{j_{1}}^{D} \otimes V_{j_{\min }}^{D} \otimes W_{j_{3}}^{0}\right) \\
& \oplus_{j_{1}, j_{2}=j_{\min }}^{j-1}\left(W_{j_{1}}^{D} \otimes W_{j_{2}}^{D} \otimes V_{j_{\min }}^{0}\right) \\
& \oplus_{j_{1}, j_{2}, j_{3}=j_{\min }}^{j-1}\left(W_{j_{1}}^{D} \otimes W_{j_{2}}^{D} \otimes W_{j_{3}}^{0}\right),
\end{aligned}
$$

we can give the definition of anisotropic divergence-free wavelets as follows.

Definition 8. For $j_{1}, j_{2}$, and $j_{3} \geq j_{\min }$, the anisotropic divergence-free wavelets are defined by

$$
\begin{aligned}
& \Psi_{\mathbf{j}, \mathbf{k}}^{\mathrm{div},(1,1)}=\frac{1}{\sqrt{4^{j_{3}}+1}} \operatorname{curl}\left[\varphi_{j_{\min }, k_{1}}^{0} \cdot \varphi_{j_{\min }, k_{2}}^{D} \cdot \psi_{j_{3}, k_{3}}^{D} \delta_{1}\right] \\
& \Psi_{\mathbf{j}, \mathbf{k}}^{\mathrm{div},(1,2)}=\frac{1}{\sqrt{4^{j_{2}}+1}} \operatorname{curl}\left[\varphi_{j_{\min }, k_{1}}^{0} \cdot \psi_{j_{2}, k_{2}}^{D} \cdot \varphi_{j_{\min }, k_{3}}^{D} \delta_{1}\right] \\
& \Psi_{\mathbf{j}, \mathbf{k}}^{\mathrm{div},(1,3)}=\frac{1}{\sqrt{2}} \operatorname{curl}\left[\psi_{j_{1}, k_{1}}^{0} \cdot \varphi_{j_{\min }, k_{2}}^{D} \cdot \varphi_{j_{\min }, k_{3}}^{D} \delta_{1}\right] \\
& \Psi_{\mathbf{j}, \mathbf{k}}^{\mathrm{div},(1,4)}=\frac{1}{\sqrt{4^{j_{2}}+4^{j_{3}}}} \operatorname{curl}\left[\varphi_{j_{\min }, k_{1}}^{0} \cdot \psi_{j_{2}, k_{2}}^{D} \cdot \psi_{j_{3}, k_{3}}^{D} \delta_{1}\right] \\
& \Psi_{\mathbf{j}, \mathbf{k}}^{\mathrm{div},(1,5)}=\frac{1}{\sqrt{4^{j_{3}}+1}} \operatorname{curl}\left[\psi_{j_{1}, k_{1}}^{0} \cdot \varphi_{j_{\min }, k_{2}}^{D} \cdot \psi_{j_{3}, k_{3}}^{D} \delta_{1}\right] \\
& \Psi_{\mathbf{j}, \mathbf{k}}^{\mathrm{div},(1,6)}=\frac{1}{\sqrt{4^{j_{2}}+1}} \operatorname{curl}\left[\psi_{j_{1}, k_{1}}^{0} \cdot \psi_{j_{2}, k_{2}}^{D} \cdot \varphi_{j_{\min }, k_{3}}^{D} \delta_{1}\right] \\
& \Psi_{\mathbf{j}, \mathbf{k}}^{\mathrm{div},(1,7)}=\frac{1}{\sqrt{4^{j_{2}}+4^{j_{3}}}} \operatorname{curl}\left[\psi_{j_{1}, k_{1}}^{0} \cdot \psi_{j_{2}, k_{2}}^{D} \cdot \psi_{j_{3}, k_{3}}^{D} \delta_{1}\right], \\
& \Psi_{\mathbf{j}, \mathbf{k}}^{\mathrm{div},(2,1)}=\frac{1}{\sqrt{4^{j_{3}}+1}} \operatorname{curl}\left[\varphi_{j_{\min }, k_{1}}^{D} \cdot \varphi_{j_{\min }, k_{2}}^{0} \cdot \psi_{j_{3}, k_{3}}^{D} \delta_{2}\right] \\
& \Psi_{\mathbf{j}, \mathbf{k}}^{\operatorname{div}(2,2)}=\frac{1}{\sqrt{2}} \operatorname{curl}\left[\varphi_{j_{\min }, k_{1}}^{D} \cdot \psi_{j_{2}, k_{2}}^{0} \cdot \varphi_{j_{\min }, k_{3}}^{D} \delta_{2}\right] \\
& \Psi_{\mathbf{j}, \mathbf{k}}^{\mathrm{div},(2,3)}=\frac{1}{\sqrt{4^{j_{1}}+1}} \operatorname{curl}\left[\psi_{j_{1}, k_{1}}^{D} \cdot \varphi_{j_{\min }, k_{2}}^{0} \cdot \varphi_{j_{\min }, k_{3}}^{D} \delta_{2}\right] \\
& \Psi_{\mathbf{j}, \mathbf{k}}^{\mathrm{div},(2,4)}=\frac{1}{\sqrt{4^{j_{3}}+1}} \operatorname{curl}\left[\varphi_{j_{\min }, k_{1}}^{D} \cdot \psi_{j_{2}, k_{2}}^{0} \cdot \psi_{j_{3}, k_{3}}^{D} \delta_{2}\right] \\
& \Psi_{\mathbf{j}, \mathbf{k}}^{\mathrm{div},(2,5)}=\frac{1}{\sqrt{4^{j_{1}}+4^{j_{3}}}} \operatorname{curl}\left[\psi_{j_{1}, k_{1}}^{D} \cdot \varphi_{j_{\min },}^{0}, k_{2} \cdot \psi_{j_{3}, k_{3}}^{D} \delta_{2}\right] \\
& \Psi_{\mathbf{j}, \mathbf{k}}^{\mathrm{div},(2,6)}=\frac{1}{\sqrt{4^{j_{1}}+1}} \operatorname{curl}\left[\psi_{j_{1}, k_{1}}^{D} \cdot \psi_{j_{2}, k_{2}}^{0} \cdot \varphi_{j_{\min }, k_{3}}^{D} \delta_{2}\right]
\end{aligned}
$$


6

Journal of Applied Mathematics

$$
\begin{aligned}
& \Psi_{\mathbf{j}, \mathbf{k}}^{\operatorname{div},(2,7)}=\frac{1}{\sqrt{4^{j_{1}}+4^{j_{3}}}} \operatorname{curl}\left[\psi_{j_{1}, k_{1}}^{D} \cdot \psi_{j_{2}, k_{2}}^{0} \cdot \psi_{j_{3}, k_{3}}^{D} \delta_{2}\right], \\
& \Psi_{\mathbf{j}, \mathbf{k}}^{\mathrm{div},(3,1)}=\frac{1}{\sqrt{2}} \operatorname{curl}\left[\varphi_{j_{\min }, k_{1}}^{D} \cdot \varphi_{j_{\min }, k_{2}}^{D} \cdot \psi_{j_{3}, k_{3}}^{0} \delta_{3}\right] \\
& \Psi_{\mathbf{j}, \mathbf{k}}^{\mathrm{div},(3,2)}=\frac{1}{\sqrt{4^{j_{2}}+1}} \operatorname{curl}\left[\varphi_{j_{\min }, k_{1}}^{D} \cdot \psi_{j_{2}, k_{2}}^{D} \cdot \varphi_{j_{\min }, k_{3}}^{0} \delta_{3}\right] \\
& \Psi_{\mathbf{j}, \mathbf{k}}^{\mathrm{div},(3,3)}=\frac{1}{\sqrt{4^{j_{1}}+1}} \operatorname{curl}\left[\psi_{j_{1}, k_{1}}^{D} \cdot \varphi_{j_{\min },}^{D}, k_{2} \cdot \varphi_{j_{\min },}^{0}, k_{3} \delta_{3}\right] \\
& \Psi_{\mathbf{j}, \mathbf{k}}^{\operatorname{div},(3,4)}=\frac{1}{\sqrt{4^{j_{2}}+1}} \operatorname{curl}\left[\varphi_{j_{\min }, k_{1}}^{D} \cdot \psi_{j_{2}, k_{2}}^{D} \cdot \psi_{j_{3}, k_{3}}^{0} \delta_{3}\right] \\
& \Psi_{\mathbf{j}, \mathbf{k}}^{\mathrm{div},(3,5)}=\frac{1}{\sqrt{4^{j_{1}}+1}} \operatorname{curl}\left[\psi_{j_{1}, k_{1}}^{D} \cdot \varphi_{j_{\min }, k_{2}}^{D} \cdot \psi_{j_{3}, k_{3}}^{0} \delta_{3}\right] \\
& \Psi_{\mathbf{j}, \mathbf{k}}^{\operatorname{div},(3,6)}=\frac{1}{\sqrt{4^{j_{1}}+4^{j_{2}}}} \operatorname{curl}\left[\psi_{j_{1}, k_{1}}^{D} \cdot \psi_{j_{2}, k_{2}}^{D} \cdot \varphi_{j_{\min }, k_{3}}^{0} \delta_{3}\right] \\
& \Psi_{\mathbf{j}, \mathbf{k}}^{\operatorname{div},(3,7)}=\frac{1}{\sqrt{4^{j_{1}}+4^{j_{2}}}} \operatorname{curl}\left[\psi_{j_{1}, k_{1}}^{D} \cdot \psi_{j_{2}, k_{2}}^{D} \cdot \psi_{j_{3}, k_{3}}^{0} \delta_{3}\right] .
\end{aligned}
$$

Remark 9. The coefficients before the operator "curl" are used to guarantee the biorthogonality in the following construetimon of dual wavelets.

Proposition 10. Defining the wavelet spaces

$$
\vec{W}_{\mathbf{j}}^{\operatorname{div},(\varepsilon, n)}=\operatorname{span}\left\{\Psi_{\mathbf{j}, \mathbf{k}}^{\operatorname{div},(\varepsilon, n)}\right\}, \quad \varepsilon=1,2,3, n=1,2, \ldots, 7
$$

then $\vec{V}_{j}^{\text {div }}=\vec{V}_{j_{\min }}^{\text {div }} \oplus_{j_{\min } \leq j_{1}, j_{2}, j_{3} \leq j-1}\left(\oplus_{\varepsilon=1,2,3, n=1,2, \ldots, 7} \vec{W}_{\mathbf{j}}^{\operatorname{div},(\varepsilon, n)}\right)$.

Proof. It can be easily obtained from (35) and Definition 8.

Definition 11. Biorthogonal divergence-free scaling functions and wavelets are defined by

$$
\begin{aligned}
& \widetilde{\Phi}_{j, \mathbf{k}}^{\mathrm{div}, 1}=\frac{1}{\sqrt{2}}\left[\widetilde{\varphi}_{j, k_{1}}^{0} \cdot \widetilde{\varphi}_{j, k_{2}}^{D} \cdot \widetilde{\gamma}_{j, k_{3}} \delta_{2}-\widetilde{\varphi}_{j, k_{1}}^{0} \cdot \widetilde{\gamma}_{j, k_{2}} \cdot \widetilde{\varphi}_{j, k_{3}}^{D} \delta_{3}\right] \\
& \widetilde{\Phi}_{j, \mathbf{k}}^{\mathrm{div}, 2}=\frac{1}{\sqrt{2}}\left[\widetilde{\gamma}_{j, k_{1}} \cdot \widetilde{\varphi}_{j, k_{2}}^{0} \cdot \widetilde{\varphi}_{j, k_{3}}^{D} \delta_{3}-\widetilde{\varphi}_{j, k_{1}}^{D} \cdot \widetilde{\varphi}_{j, k_{2}}^{0} \cdot \widetilde{\gamma}_{j, k_{3}} \delta_{1}\right] \\
& \widetilde{\Phi}_{j, \mathbf{k}}^{\mathrm{div}, 3}=\frac{1}{\sqrt{2}}\left[\widetilde{\varphi}_{j, k_{1}}^{D} \cdot \widetilde{\gamma}_{j, k_{2}} \cdot \widetilde{\varphi}_{j, k_{3}}^{0} \delta_{1}-\widetilde{\gamma}_{j, k_{1}} \cdot \widetilde{\varphi}_{j, k_{2}}^{D} \cdot \widetilde{\varphi}_{j, k_{3}}^{0} \delta_{2}\right], \\
& \widetilde{\Psi}_{\mathbf{j}, \mathbf{k}}^{\mathrm{div},(1,1)}=\frac{1}{\sqrt{4^{j_{3}}+1}}\left[2^{j_{3}} \widetilde{\varphi}_{j_{\min }, k_{1}}^{0} \cdot \widetilde{\varphi}_{j_{\min }, k_{2}}^{D} \cdot \widetilde{\psi}_{j_{3}, k_{3}, k_{1}}^{0} \cdot \delta_{2}\right. \\
& \widetilde{\Psi}_{\mathbf{j}, \mathbf{k}}^{\mathrm{div},(1,2)}=\frac{1}{\sqrt{4^{j_{2}}+1}}\left[\widetilde{\varphi}_{j_{\min },}^{0}, k_{1} \cdot \widetilde{\psi}_{j_{2}, k_{2}}^{D} \cdot \widetilde{\psi}_{j_{3}, k_{3}}^{D} \delta_{3}\right] \\
& \left.-2^{j_{2}} \widetilde{\varphi}_{j_{\min }, k_{3}}^{0} \delta_{2} \cdot \widetilde{\psi}_{j_{2}, k_{2}}^{0} \cdot \widetilde{\varphi}_{j_{\min }, k_{3}}^{D} \delta_{3}\right]
\end{aligned}
$$

$$
\begin{aligned}
& \widetilde{\Psi}_{\mathbf{j}, \mathbf{k}}^{\mathrm{div},(1,3)}=\frac{1}{\sqrt{2}}\left[\widetilde{\psi}_{j_{1}, k_{1}}^{0} \cdot \widetilde{\varphi}_{j_{\min }, k_{2}}^{D} \cdot \widetilde{\gamma}_{j_{\min }, k_{3}} \delta_{2}\right. \\
& \left.-\widetilde{\psi}_{j_{1}, k_{1}}^{0} \cdot \widetilde{\gamma}_{j_{\min }, k_{2}} \cdot \widetilde{\varphi}_{j_{\min }, k_{3}}^{D} \delta_{3}\right] \\
& \widetilde{\Psi}_{\mathbf{j}, \mathbf{k}}^{\mathrm{div},(1,4)}=\frac{1}{\sqrt{4^{j_{2}}+4^{j_{3}}}}\left[2^{j_{3}} \widetilde{\varphi}_{j_{\min }, k_{1}}^{0} \cdot \widetilde{\psi}_{j_{2}, k_{2}}^{D} \cdot \widetilde{\psi}_{j_{3}, k_{3}}^{0} \delta_{2}\right. \\
& \left.-2^{j_{2}} \widetilde{\varphi}_{j_{\min }, k_{1}}^{0} \cdot \widetilde{\psi}_{j_{2}, k_{2}}^{0} \cdot \widetilde{\psi}_{j_{3}, k_{3}}^{D} \delta_{3}\right] \\
& \widetilde{\Psi}_{\mathbf{j}, \mathbf{k}}^{\operatorname{div},(1,5)}=\frac{1}{\sqrt{4^{j_{3}}+1}}\left[2^{j_{3}} \widetilde{\psi}_{j_{1}, k_{1}}^{0} \cdot \widetilde{\varphi}_{j_{\min }, k_{2}}^{D} \cdot \widetilde{\psi}_{j_{3}, k_{3}}^{0} \delta_{2}\right. \\
& \left.-\widetilde{\psi}_{j_{1}, k_{1}}^{0} \cdot \tilde{\gamma}_{j_{\min }, k_{2}} \cdot \widetilde{\psi}_{j_{3}, k_{3}}^{D} \delta_{3}\right] \\
& \widetilde{\Psi}_{\mathbf{j}, \mathbf{k}}^{\operatorname{div},(1,6)}=\frac{1}{\sqrt{4^{j_{2}}+1}}\left[\widetilde{\psi}_{j_{1}, k_{1}}^{0} \cdot \widetilde{\psi}_{j_{2}, k_{2}}^{D} \cdot \widetilde{\gamma}_{j_{\min }, k_{3}} \delta_{2}\right. \\
& \left.-2^{j_{2}} \widetilde{\psi}_{j_{1}, k_{1}}^{0} \cdot \widetilde{\psi}_{j_{2}, k_{2}}^{0} \cdot \widetilde{\varphi}_{j_{\min }, k_{3}}^{D} \delta_{3}\right] \\
& \widetilde{\Psi}_{\mathbf{j}, \mathbf{k}}^{\operatorname{div},(1,7)}=\frac{1}{\sqrt{4^{j_{2}}+4^{j_{3}}}}\left[2^{j_{3}} \widetilde{\psi}_{j_{1}, k_{1}}^{0} \cdot \widetilde{\psi}_{j_{2}, k_{2}}^{D} \cdot \widetilde{\psi}_{j_{3}, k_{3}}^{0} \delta_{2}\right. \\
& \left.-2^{j_{2}} \widetilde{\psi}_{j_{1}, k_{1}}^{0} \cdot \widetilde{\psi}_{j_{2}, k_{2}}^{0} \cdot \widetilde{\psi}_{j_{3}, k_{3}}^{D} \delta_{3}\right], \\
& \widetilde{\Psi}_{\mathbf{j}, \mathbf{k}}^{\mathrm{div},(2,1)}=\frac{1}{\sqrt{4^{j_{3}}+1}}\left[\widetilde{\gamma}_{j_{\min }, k_{1}} \cdot \widetilde{\varphi}_{j_{\min }, k_{2}}^{0} \cdot \widetilde{\psi}_{j_{3}, k_{3}}^{D} \delta_{3}\right. \\
& \left.-2^{j_{3}} \widetilde{\varphi}_{j_{\min }, k_{1}}^{D} \cdot \widetilde{\varphi}_{j_{\min }, k_{2}}^{0} \cdot \widetilde{\psi}_{j_{3}, k_{3}}^{0} \delta_{1}\right] \\
& \widetilde{\Psi}_{\mathbf{j}, \mathbf{k}}^{\operatorname{div},(2,2)}=\frac{1}{\sqrt{2}}\left[\widetilde{\gamma}_{j_{\min }, k_{1}} \cdot \widetilde{\psi}_{j_{2}, k_{2}}^{0} \cdot \widetilde{\varphi}_{j_{\min }, k_{3}}^{D} \delta_{3}\right. \\
& \left.-\tilde{\varphi}_{j_{\min }, k_{1}}^{D} \cdot \widetilde{\psi}_{j_{2}, k_{2}}^{0} \cdot \tilde{\gamma}_{j_{\min }, k_{3}} \delta_{1}\right] \\
& \widetilde{\Psi}_{\mathbf{j}, \mathbf{k}}^{\operatorname{div},(2,3)}=\frac{1}{\sqrt{4^{j_{1}}+1}}\left[2^{j_{1}} \widetilde{\psi}_{j_{1}, k_{1}}^{0} \cdot \widetilde{\varphi}_{j_{\min }, k_{2}}^{0} \cdot \widetilde{\varphi}_{j_{\min }, k_{3}}^{D} \delta_{3}\right. \\
& \left.-\widetilde{\psi}_{j_{1}, k_{1}}^{D} \cdot \widetilde{\varphi}_{j_{\min }, k_{2}}^{0} \cdot \tilde{\gamma}_{j_{\min }, k_{3}} \delta_{1}\right] \\
& \widetilde{\Psi}_{\mathbf{j}, \mathbf{k}}^{\operatorname{div},(2,4)}=\frac{1}{\sqrt{4^{j_{3}}+1}}\left[\widetilde{\gamma}_{j_{\min }, k_{1}} \cdot \widetilde{\psi}_{j_{2}, k_{2}}^{0} \cdot \widetilde{\psi}_{j_{3}, k_{3}}^{D} \delta_{3}\right. \\
& \left.-2^{j_{3}} \widetilde{\varphi}_{j_{\min }, k_{1}}^{D} \cdot \widetilde{\psi}_{j_{2}, k_{2}}^{0} \cdot \widetilde{\psi}_{j_{3}, k_{3}}^{0} \delta_{1}\right] \\
& \widetilde{\Psi}_{\mathbf{j}, \mathbf{k}}^{\operatorname{div},(2,5)}=\frac{1}{\sqrt{4^{j_{1}}+4^{j_{3}}}}\left[2^{j_{1}} \widetilde{\psi}_{j_{1}, k_{1}}^{0} \cdot \widetilde{\varphi}_{j_{\min }, k_{2}}^{0} \cdot \widetilde{\psi}_{j_{3}, k_{3}}^{D} \delta_{3}\right. \\
& \left.-2^{j_{3}} \widetilde{\psi}_{j_{1}, k_{1}}^{D} \cdot \widetilde{\varphi}_{j_{\min }, k_{2}}^{0} \cdot \widetilde{\psi}_{j_{3}, k_{3}}^{0} \delta_{1}\right] \\
& \widetilde{\Psi}_{\mathbf{j}, \mathbf{k}}^{\operatorname{div},(2,6)}=\frac{1}{\sqrt{4^{j_{1}}+1}}\left[2^{j_{1}} \widetilde{\psi}_{j_{1}, k_{1}}^{0} \cdot \widetilde{\psi}_{j_{2}, k_{2}}^{0} \cdot \widetilde{\varphi}_{j_{\min }, k_{3}}^{D} \delta_{3}\right. \\
& \left.-\widetilde{\psi}_{j_{1}, k_{1}}^{D} \cdot \widetilde{\psi}_{j_{2}, k_{2}}^{0} \cdot \tilde{\gamma}_{j_{\min }, k_{3}} \delta_{1}\right] \\
& \widetilde{\Psi}_{\mathbf{j}, \mathbf{k}}^{\mathrm{div},(2,7)}=\frac{1}{\sqrt{4^{j_{1}}+4^{j_{3}}}}\left[2^{j_{1}} \widetilde{\psi}_{j_{1}, k_{1}}^{0} \cdot \widetilde{\psi}_{j_{2}, k_{2}}^{0} \cdot \widetilde{\psi}_{j_{3}, k_{3}}^{D} \delta_{3}\right. \\
& \left.-2^{j_{3}} \widetilde{\psi}_{j_{1}, k_{1}}^{D} \cdot \widetilde{\psi}_{j_{2}, k_{2}}^{0} \cdot \widetilde{\psi}_{j_{3}, k_{3}}^{0} \delta_{1}\right], \\
& \widetilde{\Psi}_{\mathbf{j}, \mathbf{k}}^{\mathrm{div},(3,1)}=\frac{1}{\sqrt{2}}\left[\widetilde{\varphi}_{j_{\min }, k_{1}}^{D} \cdot \widetilde{\gamma}_{j_{\min }, k_{2}} \cdot \widetilde{\psi}_{j_{3}, k_{3}}^{0} \delta_{1}\right. \\
& \left.-\tilde{\gamma}_{j_{\min }, k_{1}} \cdot \tilde{\varphi}_{j_{\min }, k_{2}}^{D} \cdot \tilde{\psi}_{j_{3}, k_{3}}^{0} \delta_{2}\right]
\end{aligned}
$$




$$
\begin{aligned}
& \widetilde{\Psi}_{\mathbf{j}, \mathbf{k}}^{\operatorname{div},(3,2)}=\frac{1}{\sqrt{4^{j_{2}}+1}}\left[2^{j_{2}} \widetilde{\varphi}_{j_{\min }, k_{1}}^{D} \cdot \widetilde{\psi}_{j_{2}, k_{2}}^{0} \cdot \widetilde{\varphi}_{j_{\min }, k_{3}}^{0} \delta_{1}\right. \\
& \left.-\tilde{\gamma}_{j_{\min }, k_{1}} \cdot \tilde{\psi}_{j_{2}, k_{2}}^{D} \cdot \widetilde{\varphi}_{j_{\min }, k_{3}}^{0} \delta_{2}\right] \\
& \widetilde{\Psi}_{\mathbf{j}, \mathbf{k}}^{\mathrm{div},(3,3)}=\frac{1}{\sqrt{4^{j_{1}}+1}}\left[\widetilde{\psi}_{j_{1}, k_{1}}^{D} \cdot \widetilde{\gamma}_{j_{\min }, k_{2}} \cdot \widetilde{\varphi}_{j_{\min }, k_{3}}^{0} \delta_{1}\right. \\
& \left.-2^{j_{1}} \widetilde{\psi}_{j_{1}, k_{1}}^{0} \cdot \widetilde{\varphi}_{j_{\min }, k_{2}}^{D} \cdot \widetilde{\varphi}_{j_{\min }, k_{3}}^{0} \delta_{2}\right] \\
& \widetilde{\Psi}_{\mathbf{j}, \mathbf{k}}^{\mathrm{div},(3,4)}=\frac{1}{\sqrt{4^{j_{2}}+1}}\left[2^{j_{2}} \widetilde{\varphi}_{j_{\min }, k_{1}}^{D} \cdot \widetilde{\psi}_{j_{2}, k_{2}}^{0} \cdot \widetilde{\psi}_{j_{3}, k_{3}}^{0} \delta_{1}\right. \\
& \left.-\tilde{\gamma}_{j_{\min }, k_{1}} \cdot \widetilde{\psi}_{j_{2}, k_{2}}^{D} \cdot \widetilde{\psi}_{j_{3}, k_{3}}^{0} \delta_{2}\right] \\
& \widetilde{\Psi}_{\mathbf{j}, \mathbf{k}}^{\operatorname{div},(3,5)}=\frac{1}{\sqrt{4^{j_{1}}+1}}\left[\widetilde{\psi}_{j_{1}, k_{1}}^{D} \cdot \widetilde{\gamma}_{j_{\min }, k_{2}} \cdot \widetilde{\psi}_{j_{3}, k_{3}}^{0} \delta_{1}\right. \\
& \left.-2^{j_{1}} \widetilde{\psi}_{j_{1}, k_{1}}^{0} \cdot \widetilde{\varphi}_{j_{\min }, k_{2}}^{D} \cdot \widetilde{\psi}_{j_{3}, k_{3}}^{0} \delta_{2}\right] \\
& \widetilde{\Psi}_{\mathbf{j}, \mathbf{k}}^{\operatorname{div},(3,6)}=\frac{1}{\sqrt{4^{j_{1}}+4^{j_{2}}}}\left[2^{j_{2}} \widetilde{\psi}_{j_{1}, k_{1}}^{D} \cdot \widetilde{\psi}_{j_{2}, k_{2}}^{0} \cdot \widetilde{\varphi}_{j_{\min }, k_{3}}^{0} \delta_{1}\right. \\
& \left.-2^{j_{1}} \widetilde{\psi}_{j_{1}, k_{1}}^{0} \cdot \widetilde{\psi}_{j_{2}, k_{2}}^{D} \cdot \widetilde{\varphi}_{j_{\min }, k_{3}}^{0} \delta_{2}\right] \\
& \widetilde{\Psi}_{\mathbf{j}, \mathbf{k}}^{\mathrm{div},(3,7)}=\frac{1}{\sqrt{4^{j_{1}}+4^{j_{2}}}}\left[2^{j_{2}} \widetilde{\psi}_{j_{1}, k_{1}}^{D} \cdot \widetilde{\psi}_{j_{2}, k_{2}}^{0} \cdot \widetilde{\psi}_{j_{3}, k_{3}}^{0} \delta_{1}\right. \\
& \left.-2^{j_{1}} \widetilde{\psi}_{j_{1}, k_{1}}^{0} \cdot \widetilde{\psi}_{j_{2}, k_{2}}^{D} \cdot \widetilde{\psi}_{j_{3}, k_{3}}^{0} \delta_{2}\right] \cdot
\end{aligned}
$$

Here, $\widetilde{\gamma}_{j, k}=-\int_{0}^{x} \widetilde{\varphi}_{j, k}^{D}(t) d t$.

Proposition 12. The families $\left\{\Phi_{j, \mathbf{k}}^{\operatorname{div}, \varepsilon}, \Psi_{\mathbf{j}, \mathbf{k}}^{\operatorname{div},(\varepsilon, n)}: j_{1}, j_{2}, j_{3} \geq\right.$ $j, \varepsilon=1,2,3, n=1,2, \ldots, 7\}$ and $\left\{\widetilde{\Phi}_{j, \mathbf{k}}^{\mathrm{div}, \varepsilon}, \widetilde{\Psi}_{\mathbf{j}, \mathbf{k}}^{\mathrm{div},(\varepsilon, n)}: j_{1}, j_{2}, j_{3} \geq\right.$ $j, \varepsilon=1,2,3, n=1,2, \ldots, 7\}$ are biorthogonal in $\left(L^{2}(\Omega)\right)^{3}$.

Proof. It is easily proved by the fact that $\widetilde{\psi}_{j, k}^{0}=$ $-2^{j} \int_{0}^{x} \widetilde{\psi}_{j, k}^{D}(t) d t$, which is shown in (16).

Theorem 13. The set $\left\{\Phi_{j_{\min }, \mathbf{k}}^{\operatorname{div}, \varepsilon}, \Psi_{\mathbf{j}, \mathbf{k}}^{\operatorname{div},(\varepsilon, n)}: j_{1}, j_{2}, j_{3} \geq j_{\min }, \varepsilon=\right.$ $1,2,3, n=1,2, \ldots, 7\}$ is a Riesz basis of $\mathscr{H}_{\mathrm{div}}(\Omega)$.

Proof. The completeness is ensured by Theorem 7 and Proposition 10. Now, it remains to prove the $L^{2}$-stability of the basis. By assumption of 1D scaling and wavelet functions, the divergence-free wavelets $\Psi_{\mathbf{j}, \mathbf{k}}^{\mathrm{div},(\varepsilon, n)}$ are compactly supported, have zero mean value, and belong to the spaces $C^{\varepsilon}$ for some $\varepsilon>0$; then they constitute a vaguelette-family ([12]). Furthermore, the Riesz stability follows from the existence of a biorthogonal wavelet family given by Proposition 12 .

\section{Curl-Free Wavelets on $[0,1]^{3}$}

The boundary condition considered in [7] is $\vec{u} \times \vec{n}=\overrightarrow{0}$ on $\Gamma=\bigcup_{k=1}^{3} \Gamma_{k}$ with

$$
\Gamma_{k}=\bigcup_{m=1, m \neq k}^{3}[0,1]^{m-1} \times\{0\} \times[0,1]^{3-m}, \quad 1 \leq k \leq 3 .
$$

It holds that $\vec{u} \times \vec{n}=\overrightarrow{0}$ on $\Gamma$ if and only if $u_{k}=0$ on $\Gamma_{k}(1 \leq k \leq 3)$, which is shown in Figure 3 .

In this section, we mainly consider the following space:

$$
\begin{aligned}
\mathscr{H}_{\text {curl }} & (\Omega) \\
= & \left\{\vec{u} \in\left(L^{2}(\Omega)\right)^{3}=: \operatorname{curl} \vec{u}=\overrightarrow{0}, \vec{u} \times \vec{n}=\overrightarrow{0} \text { on } \partial \Omega\right\}
\end{aligned}
$$

with free-slip boundary as Figure 4.

An equivalent characterization is firstly given for $\mathscr{H}_{\text {curl }}(\Omega)$; and then we will give the MRA and wavelets for it.

Proposition 14. There is the characterization $\mathscr{H}_{\text {curl }}(\Omega)=$ $\left\{\vec{u}=\operatorname{grad} \varphi: \varphi \in H_{0}^{1}(\Omega)\right\}$.

Proof. Suppose $\varphi \in H_{0}^{1}(\Omega)$; then $\vec{u}=\operatorname{grad} \varphi=\left(\partial_{1} \varphi, \partial_{2} \varphi\right.$, $\left.\partial_{3} \varphi\right)^{T} \in\left(L^{2}(\Omega)\right)^{3}$. Moreover,

$$
\begin{aligned}
\operatorname{curl} \vec{u} & =\operatorname{curl} \cdot \operatorname{grad} \varphi \\
& =\left(\partial_{2} \partial_{3} \varphi-\partial_{3} \partial_{2} \varphi, \partial_{3} \partial_{1} \varphi-\partial_{1} \partial_{3} \varphi, \partial_{1} \partial_{2} \varphi-\partial_{2} \partial_{1} \varphi\right)^{T} \\
& =\overrightarrow{0} .
\end{aligned}
$$

Note that

$$
\begin{aligned}
& \partial_{1} \varphi(x, y, 0)=\lim _{\Delta x \rightarrow 0} \frac{\varphi(x+\Delta x, y, 0)-\varphi(x, y, 0)}{\Delta x}=0, \\
& \partial_{1} \varphi(x, y, 1)=\lim _{\Delta x \rightarrow 0} \frac{\varphi(x+\Delta x, y, 1)-\varphi(x, y, 1)}{\Delta y}=0, \\
& \partial_{1} \varphi(x, 0, z)=\lim _{\Delta x \rightarrow 0} \frac{\varphi(x+\Delta x, 0, z)-\varphi(x, 0, z)}{\Delta x}=0 \\
& \partial_{1} \varphi(x, 1, z)=\lim _{\Delta x \rightarrow 0} \frac{\varphi(x+\Delta x, 1, z)-\varphi(x, 1, z)}{\Delta y}=0
\end{aligned}
$$

therefore,

$$
\begin{array}{ll}
u_{1}(x, y, 0)=u_{1}(x, y, 1)=0, & \forall 0 \leq x, y \leq 1 . \\
u_{1}(x, 0, z)=u_{1}(x, 1, z)=0, & \forall 0 \leq x, z \leq 1 .
\end{array}
$$

In the same way, one can obtain

$$
\begin{array}{ll}
u_{2}(x, y, 0)=u_{2}(x, y, 1)=0, & \forall 0 \leq x, y \leq 1, \\
u_{2}(0, y, z)=u_{2}(1, y, z)=0, & \forall 0 \leq y, z \leq 1, \\
u_{3}(0, y, z)=u_{3}(1, y, z)=0, & \forall 0 \leq y, z \leq 1, \\
u_{3}(x, 0, z)=u_{3}(x, 1, z)=0, & \forall 0 \leq x, z \leq 1 .
\end{array}
$$

This is equivalent to $\vec{u} \times \vec{n}=\overrightarrow{0}$. Therefore, $\vec{u}=\operatorname{grad} \varphi \epsilon$ $\mathscr{H}_{\text {curl }}(\Omega)$.

On the other hand, suppose $\vec{u} \in \mathscr{H}_{\text {curl }}(\Omega)$; then we will prove that there exists a function $\varphi \in H_{0}^{1}(\Omega)$, such that $\vec{u}=$ $\operatorname{grad} \varphi$. Since $\operatorname{curl} \vec{u}=\overrightarrow{0}$, then

$$
\partial_{2} u_{3}=\partial_{3} u_{2}, \quad \partial_{3} u_{1}=\partial_{1} u_{3}, \quad \partial_{1} u_{2}=\partial_{2} u_{1} \text {. }
$$



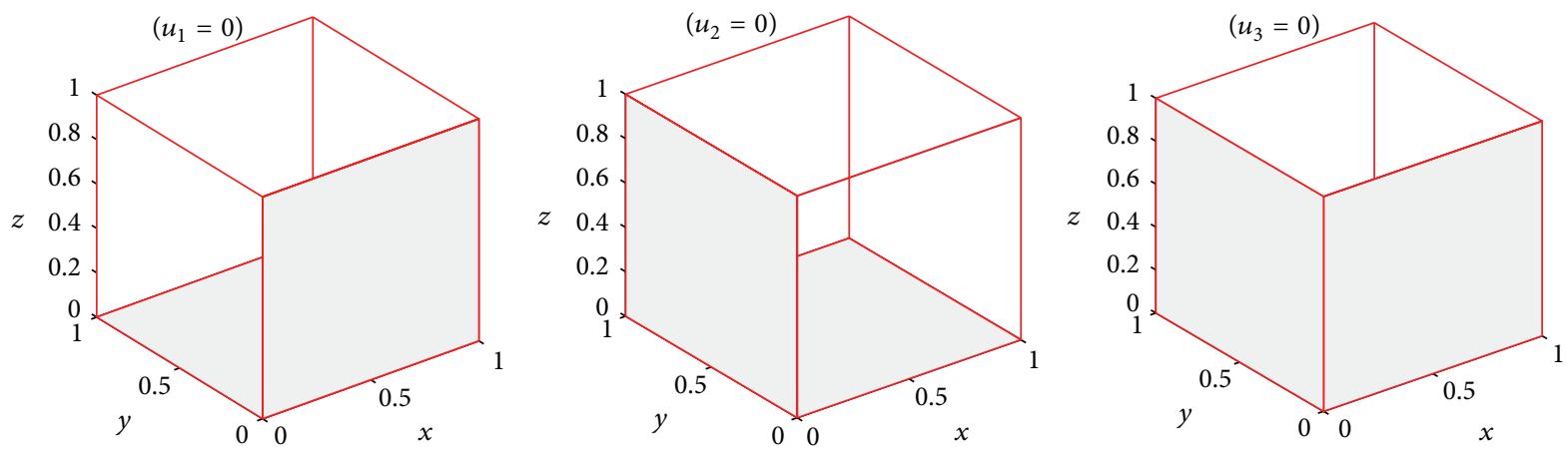

FIGURE 3: Slip boundary condition (curl).
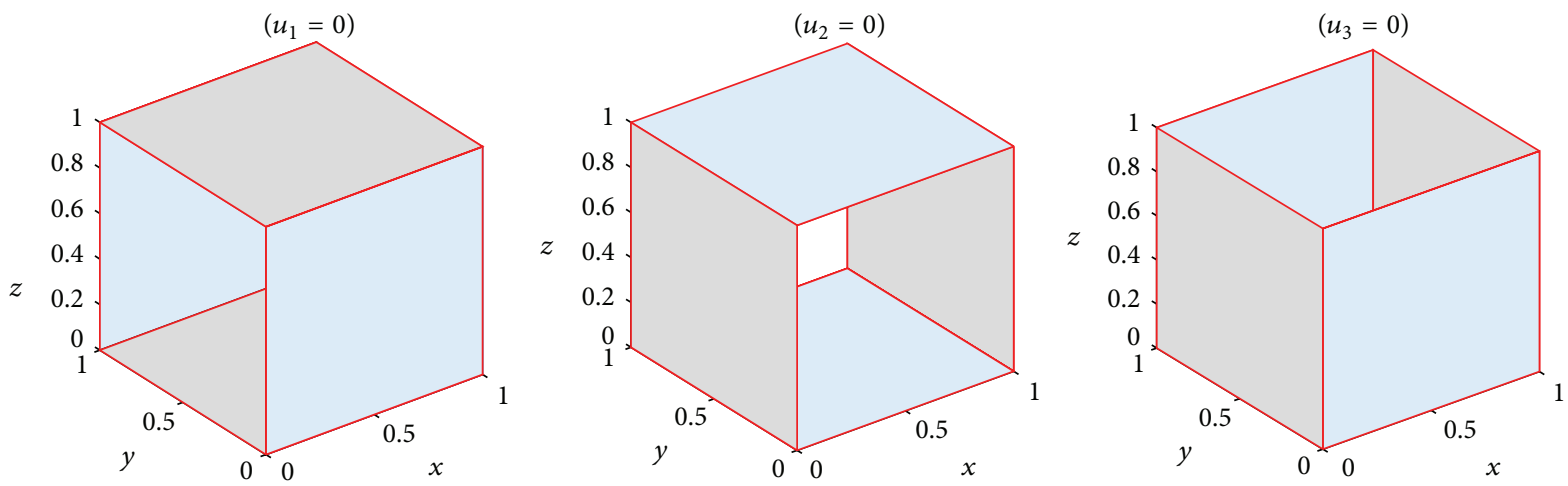

FIGURE 4: Free-slip boundary condition (curl).

By Stokes formula, there exists a primitive function $\varphi \in$ $H^{1}(\Omega)$ such that

$$
\begin{aligned}
& d \varphi(x, y, z) \\
& \quad=u_{1}(x, y, z) d x+u_{2}(x, y, z) d y+u_{3}(x, y, z) d z .
\end{aligned}
$$

Therefore, $\partial \varphi / \partial x=u_{1}, \partial \varphi / \partial y=u_{2}$, and $\partial \varphi / \partial z=u_{3}$; that is $\vec{u}=\operatorname{grad} \varphi$. Furthermore, $\vec{u} \times \vec{n}=\overrightarrow{0}$ means that

$$
\begin{array}{ll}
u_{1}(x, y, 0)=u_{1}(x, y, 1)=0, & \forall 0 \leq x, y \leq 1, \\
u_{2}(0, y, z)=u_{2}(1, y, z)=0, & \forall 0 \leq y, z \leq 1, \\
u_{3}(x, 0, z)=u_{3}(x, 1, z)=0, & \forall 0 \leq x, z \leq 1 .
\end{array}
$$

Noting that

$$
\begin{aligned}
\varphi(x, y, z) & =\int_{x_{0}}^{x} u_{1}(r, y, z) d r=\int_{y_{0}}^{y} u_{2}(x, s, z) d s \\
& =\int_{z_{0}}^{z} u_{3}(x, y, t) d t,
\end{aligned}
$$

we obtain $\varphi(x, y, 0)=\varphi(x, y, 1)=0, \varphi(0, y, z)=\varphi(1, y, z)=$ 0 , and $\varphi(x, 0, z)=\varphi(x, 1, z)=0$. Therefore, $\varphi \in H_{0}^{1}(\Omega)$.

Noting that $V_{j}^{D} \otimes V_{j}^{D} \otimes V_{j}^{D}$ is an MRA of $\left(H_{0}^{1}(\Omega)\right)^{3}$, we give the following definition.
Definition 15. For $j \geq j_{\min }$, the curl-free scaling function spaces $\vec{V}_{j}^{\text {curl }}$ are defined by

$$
\vec{V}_{j}^{\text {curl }}=\operatorname{grad}\left(V_{j}^{D} \otimes V_{j}^{D} \otimes V_{j}^{D}\right)=\operatorname{span}\left\{\Phi_{j, \mathbf{k}}^{\text {curl }}\right\},
$$

where the curl-free scaling functions are given by

$$
\begin{gathered}
\Phi_{j, \mathbf{k}}^{\text {curl }}=: \frac{1}{\sqrt{3}} \operatorname{grad}\left(\varphi_{j, k_{1}}^{D} \cdot \varphi_{j, k_{2}}^{D} \cdot \varphi_{j, k_{3}}^{D}\right) \\
=\frac{1}{\sqrt{3}}\left(\left(\varphi_{j, k_{1}}^{D}\right)^{\prime} \cdot \varphi_{j, k_{2}}^{D} \cdot \varphi_{j, k_{3}}^{D}, \varphi_{j, k_{1}}^{D} \cdot\left(\varphi_{j, k_{2}}^{D}\right)^{\prime}\right. \\
\left.\cdot \varphi_{j, k_{3}}^{D}, \varphi_{j, k_{1}}^{D} \cdot \varphi_{j, k_{2}}^{D} \cdot\left(\varphi_{j, k_{3}}^{D}\right)^{\prime}\right)^{T} .
\end{gathered}
$$

For convenience, we also consider the standard MRA $\vec{V}_{j}$ of $\left(L^{2}(\Omega)\right)^{3}$ :

$$
\vec{V}_{j}=\left(V_{j}^{0} \otimes V_{j}^{1} \otimes V_{j}^{1}\right) \times\left(V_{j}^{1} \otimes V_{j}^{0} \otimes V_{1}^{1}\right) \times\left(V_{j}^{1} \otimes V_{j}^{1} \otimes V_{j}^{0}\right) .
$$

Theorem 16. The curl-free scaling function spaces $\left\{\vec{V}_{j}^{\text {curl }}\right\}_{j \geq j_{\min }}$ are a multiresolution analysis of $\mathscr{H}_{\text {curl }}(\Omega)$.

Proof. Since $\mathscr{H}_{\text {curl }}(\Omega) \cap \vec{V}_{j}$ is a multiresolution analysis of $\mathscr{H}_{\text {curl }}(\Omega)$, it is reduced to prove

$$
\vec{V}_{j}^{\text {curl }}=\mathscr{H}_{\text {curl }}(\Omega) \cap \vec{V}_{j} .
$$


Noting that $(d / d x) V_{j}^{1}=V_{j}^{0}$ and $V_{j}^{D} \subseteq V_{j}^{1}$, we know $\vec{V}_{j}^{\text {curl }} \subset \vec{V}_{j}$. Furthermore, $\vec{V}_{j}^{\text {curl }} \subset \mathscr{H}_{\text {curl }}(\Omega)$ by construction. Therefore, $\vec{V}_{j}^{\text {curl }} \subset \mathscr{H}_{\text {curl }}(\Omega) \cap \vec{V}_{j}$.

Conversely, let $\vec{u} \in \mathscr{H}_{\text {curl }}(\Omega) \cap \vec{V}_{j}$, we are going to prove $\vec{u} \in \vec{V}_{j}^{\text {curl }}$. Let $\vec{P}_{j}$ be the biorthogonal projector on $\vec{V}_{j}$. On the one hand, since $\vec{u} \in \vec{V}_{j}$, we have $\vec{u}=\vec{P}_{j} \vec{u}$. On the other hand, since $\vec{u} \in \mathscr{H}_{\text {curl }}(\Omega)$, there exists a $\varphi \in H_{0}^{1}(\Omega)$ such that $\vec{u}=$ $\operatorname{grad} \varphi$. Thus,

$$
\vec{u}=\vec{P}_{j}[\operatorname{grad} \varphi] .
$$

Since $\left(V_{j}^{D} \otimes V_{j}^{D} \otimes V_{j}^{D}\right)_{j \geq j_{\min }}$ forms an MRA of $H_{0}^{1}(\Omega)$, we can decompose $\varphi$ as

$$
\varphi=P_{j}^{D}(\varphi)+\sum_{j_{1}, j_{2}, j_{3} \geq j} \sum_{n=1}^{7} Q_{n, J}^{D}(\varphi), \quad J=\left(j_{1}, j_{2}, j_{3}\right),
$$

where

$$
\begin{aligned}
& P_{j}^{D}(\varphi)=\sum_{\mathbf{k}} \mathcal{c}_{\mathbf{k}} \varphi_{j, k_{1}}^{D} \varphi_{j, k_{2}}^{D} \varphi_{j, k_{3}}^{D}, \\
& Q_{1, J}^{D}(\varphi)=\sum_{j_{3} \geq j} \sum_{\mathbf{k}} d_{j_{3}, \mathbf{k}}^{1} \varphi_{j, k_{1}}^{D} \varphi_{j, k_{2}}^{D} \psi_{j_{3}, k_{3}}^{D}, \\
& Q_{2, J}^{D}(\varphi)=\sum_{j_{2} \geq j} \sum_{\mathbf{k}} d_{j_{2}, \mathbf{k}}^{2} \varphi_{j, k_{1}}^{D} \psi_{j_{2}, k_{2}}^{D} \varphi_{j, k_{3}}^{D}, \\
& Q_{3, J}^{D}(\varphi)=\sum_{j_{1} \geq j} \sum_{\mathbf{k}} d_{j_{1}, \mathbf{k}}^{3} \psi_{j_{1}, k_{1}}^{D} \varphi_{j, k_{2}}^{D} \varphi_{j, k_{3}}^{D}, \\
& Q_{4, J}^{D}(\varphi)=\sum_{j_{2}, j_{3} \geq j} \sum_{\mathbf{k}} d_{j_{2}, j_{3}, \mathbf{k}}^{4} \varphi_{j, k_{1}}^{D} \psi_{j_{2}, k_{2}}^{D} \psi_{j_{3}, k_{3}}^{D}, \\
& Q_{5, J}^{D}(\varphi)=\sum_{j_{1}, j_{3} \geq j} \sum_{\mathbf{k}} d_{j_{1}, j_{3}, \mathbf{k}}^{5} \psi_{j_{1}, k_{1}}^{D} \varphi_{j, k_{2}}^{D} \psi_{j_{3}, k_{3}}^{D}, \\
& Q_{6, J}^{D}(\varphi)=\sum_{j_{1}, j_{2} \geq j} \sum_{\mathbf{k}} d_{j_{1}, j_{2}, \mathbf{k}}^{6} \psi_{j_{1}, k_{1}}^{D} \psi_{j_{2}, k_{2}}^{D} \varphi_{j, k_{3}}^{D}, \\
& Q_{7, J}^{D}(\varphi)=\sum_{j_{1}, j_{2}, j_{3} \geq j} \sum_{\mathbf{k}} d_{j_{1}, j_{2}, j_{3}, \mathbf{k}}^{7} \psi_{j_{1}, k_{1}}^{D} \psi_{j_{2}, k_{2}}^{D} \psi_{j_{3}, k_{3}}^{D}
\end{aligned}
$$

are the biorthogonal projectors on, respectively, $V_{j}^{D} \otimes V_{j}^{D} \otimes V_{j}^{D}$, $V_{j}^{D} \otimes V_{j}^{D} \otimes W_{j_{3}}^{D}, V_{j}^{D} \otimes W_{j_{2}}^{D} \otimes V_{j}^{D}, W_{j_{1}}^{D} \otimes V_{j}^{D} \otimes V_{j}^{D}, V_{j}^{D} \otimes W_{j_{2}}^{D} \otimes W_{j_{3}}^{D}$, $W_{j_{1}}^{D} \otimes V_{j}^{D} \otimes W_{j_{3}}^{D}, W_{j_{1}}^{D} \otimes W_{j_{2}}^{D} \otimes V_{j}^{D}$, and $W_{j_{1}}^{D} \otimes W_{j_{2}}^{D} \otimes W_{j_{3}}^{D}$.

Noting that

$$
\begin{aligned}
\operatorname{grad} & \left(\varphi_{j, k_{1}}^{D} \varphi_{j, k_{2}}^{D} \psi_{j_{3}, k_{3}}^{D}\right) \in\left(V_{j}^{0} \otimes V_{j}^{D} \otimes W_{j_{3}}^{D}\right) \\
& \times\left(V_{j}^{D} \otimes V_{j}^{0} \otimes W_{j_{3}}^{D}\right) \times\left(V_{j}^{D} \otimes V_{j}^{D} \otimes W_{j_{3}}^{0}\right),
\end{aligned}
$$

then $\vec{P}_{j}\left[\operatorname{grad} Q_{1, J}^{D}(\varphi)\right]=\overrightarrow{0}$. Similarly, $\vec{P}_{j}\left[\operatorname{grad} Q_{n, J}^{D}(\varphi)\right]=\overrightarrow{0}$ for $2 \leq n \leq 7$. Therefore,

$$
\vec{u}=\vec{P}_{j}[\operatorname{grad} \varphi]=\vec{P}_{j}\left[\operatorname{grad} P_{j}^{D}(\varphi)\right] .
$$

Since $\operatorname{grad} P_{j}^{D}(\varphi) \in \vec{V}_{j}^{\text {curl }} \subset \vec{V}_{j}$, then we obtain

$$
\vec{u}=\operatorname{grad} P_{j}^{D}(\varphi) \in \vec{V}_{j}^{\text {curl }} .
$$

Definition 17. For $j_{1}, j_{2}$, and $j_{3} \geq j_{\min }$, the anisotropic curlfree wavelets and wavelet spaces are defined by

$$
\begin{aligned}
& \Psi_{\mathbf{j}, \mathbf{k}}^{\text {curl, } 1}=\frac{1}{\sqrt{4^{j_{3}}+2}} \operatorname{grad}\left[\varphi_{j_{\min }, k_{1}}^{D} \cdot \varphi_{j_{\min }}^{D}, k_{2} \cdot \psi_{j_{3}, k_{3}}^{D}\right] \\
& \Psi_{\mathbf{j}, \mathbf{k}}^{\text {curl }, 2}=\frac{1}{\sqrt{4^{j_{2}}+2}} \operatorname{grad}\left[\varphi_{j_{\min }, k_{1}}^{D} \cdot \psi_{j_{2}, k_{2}}^{D} \cdot \varphi_{j_{\min }, k_{3}}^{D}\right] \\
& \Psi_{\mathbf{j}, \mathbf{k}}^{\text {curl,3 }}=\frac{1}{\sqrt{4^{j_{1}}+2}} \operatorname{grad}\left[\psi_{j_{1}, k_{1}}^{D} \cdot \varphi_{j_{\min }, k_{2}}^{D} \cdot \varphi_{j_{\min }, k_{3}}^{D}\right] \\
& \Psi_{\mathbf{j}, \mathbf{k}}^{\text {curl, } 4}=\frac{1}{\sqrt{4^{j_{2}}+4^{j_{3}}+1}} \operatorname{grad}\left[\varphi_{j_{\min }, k_{1}}^{D} \cdot \psi_{j_{2}, k_{2}}^{D} \cdot \psi_{j_{3}, k_{3}}^{D}\right] \\
& \Psi_{\mathbf{j}, \mathbf{k}}^{\text {curl, }, 5}=\frac{1}{\sqrt{4^{j_{1}}+4^{j_{3}}+1}} \operatorname{grad}\left[\psi_{j_{1}, k_{1}}^{D} \cdot \varphi_{j_{\min }, k_{2}}^{D} \cdot \psi_{j_{3}, k_{3}}^{D}\right] \\
& \Psi_{\mathbf{j}, \mathbf{k}}^{\text {curl, } 6}=\frac{1}{\sqrt{4^{j_{1}}+4^{j_{2}}+1}} \operatorname{grad}\left[\psi_{j_{1}, k_{1}}^{D} \cdot \psi_{j_{2}, k_{2}}^{D} \cdot \varphi_{j_{\min }, k_{3}}^{D}\right] \\
& \Psi_{\mathbf{j}, \mathbf{k}}^{\text {curl, } 7}=\frac{1}{\sqrt{4^{j_{1}}+4^{j_{2}}+4^{j_{3}}}} \operatorname{grad}\left[\psi_{j_{1}, k_{1}}^{D} \cdot \psi_{j_{2}, k_{2}}^{D} \cdot \psi_{j_{3}, k_{3}}^{D}\right] .
\end{aligned}
$$

Proposition 18. Defining the wavelet spaces $\vec{W}_{\mathbf{j}}^{\mathrm{curl}, n}=$ $\operatorname{span}\left\{\Psi_{\mathbf{j}, \mathbf{k}}^{\mathrm{cur}, n}\right\}$ for $n=1,2, \ldots, 7$, then

$$
\vec{V}_{j}^{\text {curl }}=\vec{V}_{j_{\min }}^{\text {curl }} \oplus_{j_{\min } \leq j_{1}, j_{2}, j_{3} \leq j-1}\left(\oplus_{\varepsilon=1,2, \ldots, 7} \vec{W}_{\mathbf{j}}^{\mathrm{curl}, \varepsilon}\right) .
$$

Proof. The result follows from the following fact:

$$
\begin{aligned}
V_{j}^{D} \otimes V_{j}^{D} \otimes V_{j}^{D}= & \left(V_{j_{\min }}^{D} \oplus_{j_{1}=j_{\min }}^{j-1} W_{j_{1}}^{D}\right) \\
& \otimes\left(V_{j_{\min }}^{D} \Theta_{j_{2}=j_{\min }}^{j-1} W_{j_{2}}^{D}\right) \\
& \otimes\left(V_{j_{\min }}^{D} \oplus_{j_{3}=j_{\min }-1}^{j-1} W_{j_{3}}^{D}\right) \\
= & \left(V_{j_{\min }}^{D} \otimes V_{j_{\min }}^{D} \otimes V_{j_{\min }}^{D}\right) \\
& \oplus_{j_{3}=j_{\min }}^{j-1}\left(V_{j_{\min }}^{D} \otimes V_{j_{\min }}^{D} \otimes W_{j_{3}}^{D}\right) \\
& \oplus_{j_{2}=j_{\min }}^{j-1}\left(V_{j_{\min }}^{D} \otimes W_{j_{2}}^{D} \otimes V_{j_{\min }}^{D}\right) \\
& \oplus_{j_{1}=j_{\min }}^{j-1}\left(W_{j_{1}}^{D} \otimes V_{j_{\min }}^{D} \otimes V_{j_{\min }}^{D}\right) \\
& \oplus_{j_{2}, j_{3}=j_{\min }}^{j-1}\left(V_{j_{\min }}^{D} \otimes W_{j_{2}}^{D} \otimes W_{j_{3}}^{D}\right) \\
& \oplus_{j_{1}, j_{3}=j_{\min }}^{j-1}\left(W_{j_{1}}^{D} \otimes V_{j_{\min }}^{D} \otimes W_{j_{3}}^{D}\right) \\
& \oplus_{j_{1}, j_{2}=j_{\min }}^{j-1}\left(W_{j_{1}}^{D} \otimes W_{j_{2}}^{D} \otimes V_{j_{\min }}^{D}\right) \\
& \oplus_{j_{1}, j_{2}, j_{3}=j_{\min }}^{j-1}\left(W_{j_{1}}^{\mathrm{D}} \otimes W_{j_{2}}^{D} \otimes W_{j_{3}}^{D}\right) .
\end{aligned}
$$

Definition 19. Biorthogonal curl-free scaling functions and wavelets are defined by

$$
\begin{gathered}
\widetilde{\Phi}_{j, \mathbf{k}}^{\text {curl }}=\frac{1}{\sqrt{3}}\left[\widetilde{\gamma}_{j, k_{1}} \cdot \widetilde{\varphi}_{j, k_{2}}^{D} \cdot \widetilde{\varphi}_{j, k_{3}}^{D} \delta_{1}+\widetilde{\varphi}_{j, k_{1}}^{D} \cdot \widetilde{\gamma}_{j, k_{2}} \cdot \widetilde{\varphi}_{j, k_{3}}^{D} \delta_{2}\right. \\
\left.+\widetilde{\varphi}_{j, k_{1}}^{D} \cdot \widetilde{\varphi}_{j, k_{2}}^{D} \cdot \widetilde{\gamma}_{j, k_{3}} \delta_{3}\right],
\end{gathered}
$$




$$
\begin{aligned}
& \widetilde{\Psi}_{\mathbf{j}, \mathbf{k}}^{\text {curl, } 1}=\frac{1}{\sqrt{4^{j_{3}}+2}}\left[\widetilde{\gamma}_{j_{\min }, k_{1}} \widetilde{\varphi}_{j_{\min }, k_{2}}^{D} \widetilde{\psi}_{j_{3}, k_{3}}^{D} \delta_{1}\right. \\
& +\widetilde{\varphi}_{j_{\min }, k_{1}}^{D} \widetilde{\gamma}_{j_{\min }, k_{2}} \widetilde{\psi}_{j_{3}, k_{3}}^{D} \delta_{2} \\
& \left.+2^{j_{3}} \tilde{\varphi}_{j_{\min }, k_{1}}^{D} \widetilde{\varphi}_{j_{\min }, k_{2}}^{D} \widetilde{\psi}_{j_{3}, k_{3}}^{0} \delta_{3}\right], \\
& \widetilde{\Psi}_{\mathbf{j}, \mathbf{k}}^{\mathrm{curl}, 2}=\frac{1}{\sqrt{4^{j_{2}}+2}}\left[\widetilde{\gamma}_{j_{\min }, k_{1}} \widetilde{\psi}_{j_{2}, k_{2}}^{D} \widetilde{\varphi}_{j_{\min }, k_{3}}^{D} \delta_{1}\right. \\
& +2^{j_{2}} \widetilde{\varphi}_{j_{\min }, k_{1}}^{D} \widetilde{\psi}_{j_{2}, k_{2}}^{0} \widetilde{\varphi}_{j_{\min }, k_{3}}^{D} \delta_{2} \\
& \left.+\widetilde{\varphi}_{j_{\min }, k_{1}}^{D} \widetilde{\psi}_{j_{2}, k_{2}}^{D} \tilde{\gamma}_{j_{\min }, k_{3}} \delta_{3}\right] \text {, } \\
& \widetilde{\Psi}_{\mathbf{j}, \mathbf{k}}^{\text {curl,3 }}=\frac{1}{\sqrt{4^{j_{1}}+2}}\left[2^{j_{1}} \widetilde{\psi}_{j_{1}, k_{1}}^{0} \widetilde{\varphi}_{j_{\min }, k_{2}}^{D} \widetilde{\varphi}_{j_{\min }, k_{3}}^{D} \delta_{1}\right. \\
& +\widetilde{\psi}_{j_{1}, k_{1}}^{D} \widetilde{\gamma}_{j_{\min }, k_{2}} \widetilde{\varphi}_{j_{\min }, k_{3}}^{D} \delta_{2} \\
& \left.+\widetilde{\psi}_{j_{1}, k_{1}}^{D} \tilde{\varphi}_{j_{\min }, k_{2}}^{D} \tilde{\gamma}_{j_{\min }, k_{3}} \delta_{3}\right] \text {, } \\
& \widetilde{\Psi}_{\mathbf{j}, \mathbf{k}}^{\text {curl, } 4}=\frac{1}{\sqrt{4^{j_{2}}+4^{j_{3}}+1}}\left[\widetilde{\gamma}_{j_{\min }, k_{1}} \widetilde{\psi}_{j_{2}, k_{2}}^{D} \widetilde{\psi}_{j_{3}, k_{3}}^{D} \delta_{1}\right. \\
& +2^{j_{2}} \widetilde{\varphi}_{j_{\min }, k_{1}}^{D} \widetilde{\psi}_{j_{2}, k_{2}}^{0} \widetilde{\psi}_{j_{3}, k_{3}}^{D} \delta_{2} \\
& \left.+2^{j_{3}} \widetilde{\varphi}_{j_{\min }, k_{1}}^{D} \widetilde{\psi}_{j_{2}, k_{2}}^{D} \widetilde{\psi}_{j_{3}, k_{3}}^{0} \delta_{3}\right] \text {, } \\
& \widetilde{\Psi}_{\mathbf{j}, \mathbf{k}}^{\text {curl, }}=\frac{1}{\sqrt{4^{j_{1}}+4^{j_{3}}+1}}\left[2^{j_{1}} \widetilde{\psi}_{j_{1}, k_{1}}^{0} \widetilde{\varphi}_{j_{\min }, k_{2}}^{D} \widetilde{\psi}_{j_{3}, k_{3}}^{D} \delta_{1}\right. \\
& +\widetilde{\psi}_{j_{1}, k_{1}}^{D} \tilde{\gamma}_{j_{\min }, k_{2}} \widetilde{\psi}_{j_{3}, k_{3}}^{D} \delta_{2} \\
& \left.+2^{j_{3}} \widetilde{\psi}_{j_{1}, k_{1}}^{D} \widetilde{\varphi}_{j_{\min }, k_{2}}^{D} \widetilde{\psi}_{j_{3}, k_{3}}^{0} \delta_{3}\right] \text {, } \\
& \widetilde{\Psi}_{\mathbf{j}, \mathbf{k}}^{\text {curl, } 6}=\frac{1}{\sqrt{4^{j_{1}}+4^{j_{2}}+1}}\left[2^{j_{1}} \widetilde{\psi}_{j_{1}, k_{1}}^{0} \widetilde{\psi}_{j_{2}, k_{2}}^{D} \widetilde{\varphi}_{j_{\min }, k_{3}}^{D} \delta_{1}\right. \\
& +2^{j_{2}} \widetilde{\psi}_{j_{1}, k_{1}}^{D} \widetilde{\psi}_{j_{2}, k_{2}}^{0} \widetilde{\varphi}_{j_{\min }, k_{3}}^{D} \delta_{2} \\
& \left.+\tilde{\psi}_{j_{1}, k_{1}}^{D} \tilde{\psi}_{j_{2}, k_{2}}^{D} \tilde{\gamma}_{j_{\min }, k_{3}} \delta_{3}\right] \text {, } \\
& \widetilde{\Psi}_{\mathbf{j}, \mathbf{k}}^{\text {curl, } 7}=\frac{1}{\sqrt{4^{j_{1}}+4^{j_{2}}+4^{j_{3}}}}\left[2^{j_{1}} \widetilde{\psi}_{j_{1}, k_{1}}^{0} \widetilde{\psi}_{j_{2}, k_{2}}^{D} \widetilde{\psi}_{j_{3}, k_{3}}^{D} \delta_{1}\right. \\
& +2^{j_{2}} \widetilde{\psi}_{j_{1}, k_{1}}^{D} \widetilde{\psi}_{j_{2}, k_{2}}^{0} \widetilde{\psi}_{j_{3}, k_{3}}^{D} \delta_{2} \\
& \left.+2^{j_{3}} \widetilde{\psi}_{j_{1}, k_{1}}^{D} \widetilde{\psi}_{j_{2}, k_{2}}^{D} \widetilde{\psi}_{j_{3}, k_{3}}^{0} \delta_{3}\right] \text {. }
\end{aligned}
$$

Here, $\tilde{\gamma}_{j, k}$ is defined as in Definition 11.

Proposition 20. The families $\left\{\Phi_{j, \mathbf{k}}^{\mathrm{curl}}, \Psi_{\mathbf{j}, \mathbf{k}}^{\mathrm{curl}, \varepsilon}: j_{1}, j_{2}, j_{3} \geq j, \varepsilon=\right.$ $1,2, \ldots, 7\}$ and $\left\{\widetilde{\Phi}_{j, \mathbf{k}}^{\text {curl }}, \widetilde{\Psi}_{\mathbf{j}, \mathbf{k}}^{\text {curl, } \varepsilon}: j_{1}, j_{2}, j_{3} \geq j, \varepsilon=1,2, \ldots, 7\right\}$ are biorthogonal in $\left(L^{2}(\Omega)\right)^{3}$.

Theorem 21. The set $\left\{\Phi_{j_{\min }, \mathbf{k}}^{\mathrm{curl}}, \Psi_{\mathbf{j}, \mathbf{k}}^{\mathrm{curl}, \varepsilon}: j_{1}, j_{2}, j_{3} \geq j_{\min }, \varepsilon=\right.$ $1,2, \ldots, 7\}$ is a Riesz basis of $\mathscr{H}_{\text {curl }}(\Omega)$.

Proof. It can be proved by the same method as Theorem 13 .

\section{Conflict of Interests}

The authors declare that there is no conflict of interests regarding the publication of this paper.

\section{Acknowledgments}

The project is supported by the National Natural Science Foundation of China (nos. 11201094 and 11161014), Guangxi Natural Science Foundation (no. 2013GXNSFAA019330) and the fund of Education Department of Guangxi (nos. 201012MS094 and 201102ZD015).

\section{References}

[1] E. Deriaz and V. Perrier, "Divergence-free and curl-free wavelets in two dimensions and three dimensions: application to turbulent flows," Journal of Turbulence, vol. 7, no. 3, 37 pages, 2006.

[2] E. Deriaz and V. Perrier, "Orthogonal Helmholtz decomposition in arbitrary dimension using divergence-free and curl-free wavelets," Applied and Computational Harmonic Analysis, vol. 26, no. 2, pp. 249-269, 2009.

[3] Y. C. Jiang and Y. Liu, "Interpolatory curl-free wavelets and applications," International Journal of Wavelets, Multiresolution and Information Processing, vol. 5, no. 5, pp. 843-858, 2007.

[4] K. Urban, "Wavelet bases in H(div) and H(curl)," Mathematics of Computation, vol. 70, no. 234, pp. 739-766, 2001.

[5] Y. C. Jiang, "Interpolatory curl-free wavelete on bounded domains and characterization of Besov spaces," Journal of Inequalities and Applications, vol. 68, pp. 1-13, 2012.

[6] R. Stevenson, "Divergence-free wavelet bases on the hypercube," Applied and Computational Harmonic Analysis, vol. 30, no. 1, pp. 1-19, 2011.

[7] Y. C. Jiang, "Anisotropic curl-free wavelet bases on the unit cube," Acta Mathematica Sinica, vol. 29, no. 4, pp. 801-814, 2013.

[8] R. Stevenson, "Divergence-free wavelet bases on the hypercube: free-slip boundary conditions, and applications for solving the instationary Stokes equations," Mathematics of Computation, vol. 80, no. 275, pp. 1499-1523, 2011.

[9] Y. C. Jiang, "Anisotropic curl-free wavelets with boundary conditions," Journal of Inequalities and Applications, vol. 205, pp. 110, 2012.

[10] S. K. Harouna and V. Perrier, "Effective construction of divergence-free wavelets on the square," Journal of Computational and Applied Mathematics, vol. 240, pp. 74-86, 2013.

[11] P. G. Lemarie-Rieusset, "Analyses multi-résolutions non orthogonales, commutation entre projecteurs et dérivation et ondelettes vecteurs à divergence nulle," Revista Matemática Iberoamericana, vol. 8, no. 2, pp. 221-237, 1992.

[12] A. Jouini and P. G. Lemarié-Rieusset, "Analyse multi-résolution bi-orthogonale sur l'intervalle et applications," Annales de l'Institut Henri Poincaré. Analyse Non Linéaire, vol. 10, no. 4, pp. 453-476, 1993.

[13] P. Monasse and V. Perrier, "Orthonormal wavelet bases adapted for partial differential equations with boundary conditions," SIAM Journal on Mathematical Analysis, vol. 29, no. 4, pp. 10401065, 1998.

[14] C. Amrouche, C. Bernardi, M. Dauge, and V. Girault, "Vector potentials in three-dimensional non-smooth domains," Mathematical Methods in the Applied Sciences, vol. 21, no. 9, pp. 823864, 1998. 


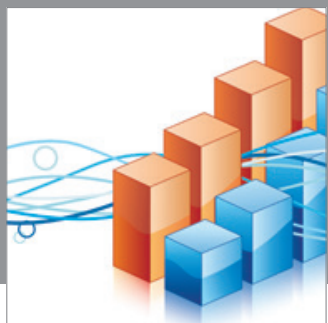

Advances in

Operations Research

mansans

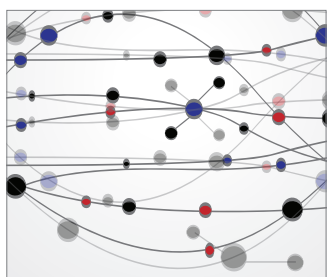

The Scientific World Journal
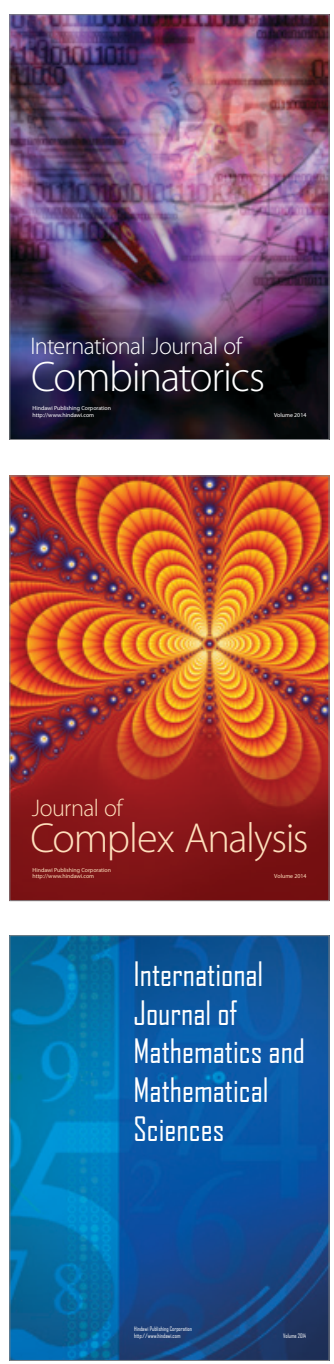
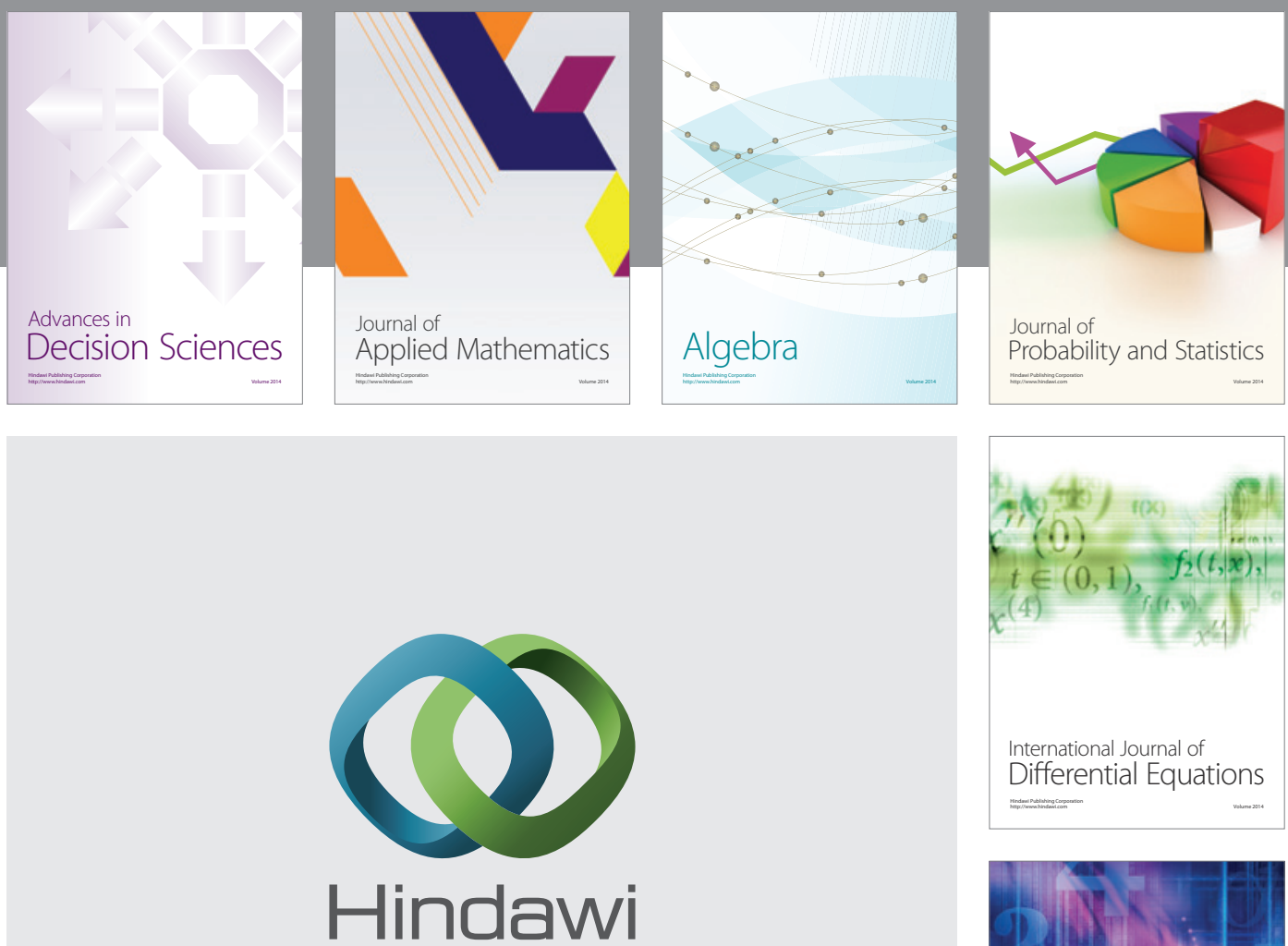

Submit your manuscripts at http://www.hindawi.com
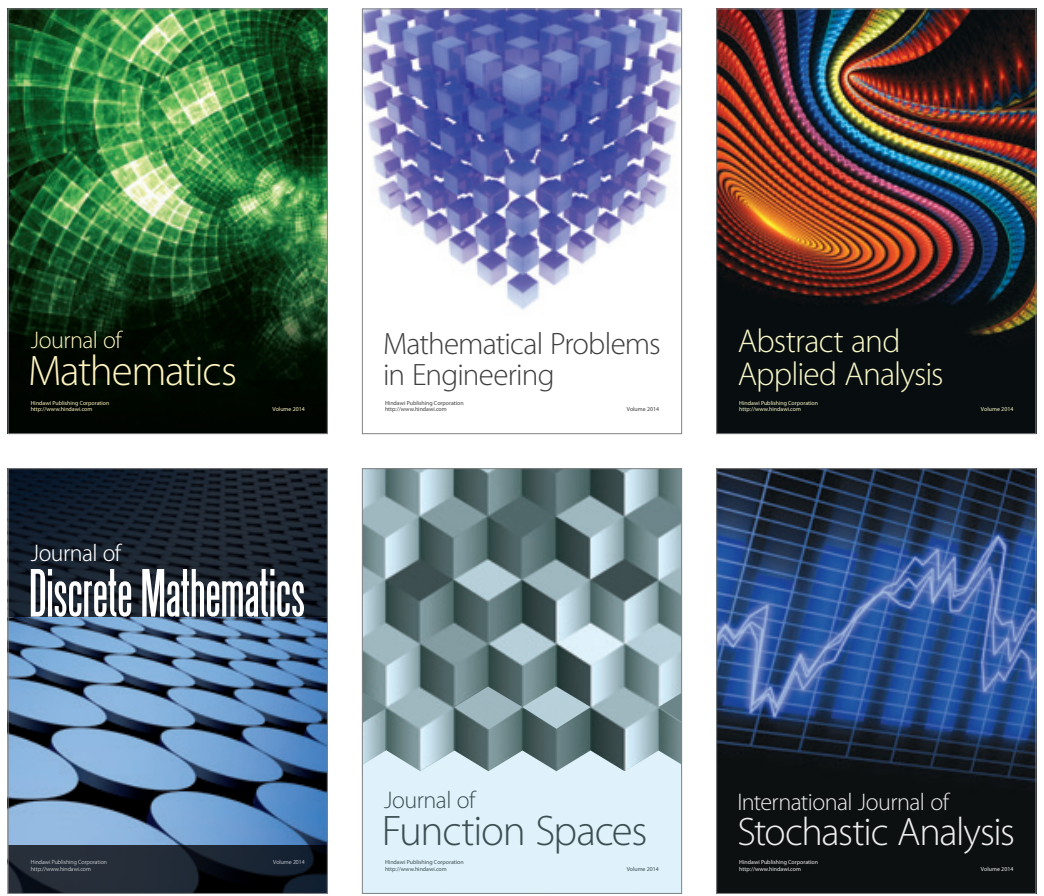

Journal of

Function Spaces

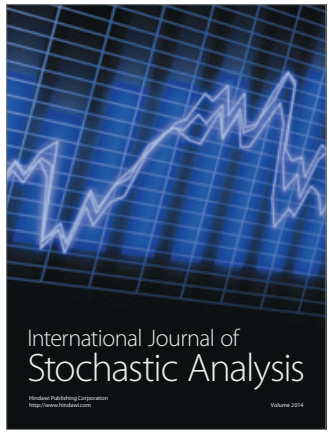

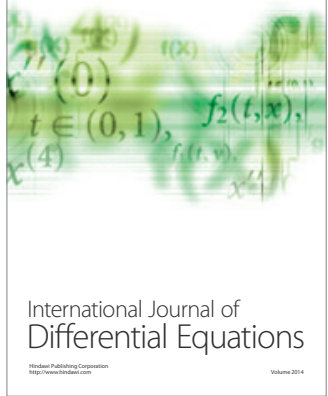
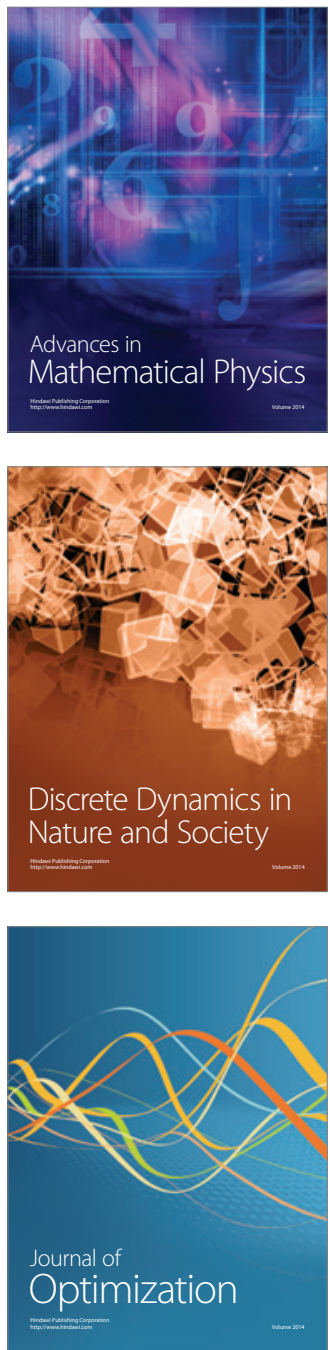